\title{
Marker-Based, 3-D Adaptive Cartesian Grid Method for Multiphase Flow around Irregular Geometries
}

\author{
Eray Uzgoren* \\ Virginia Polytechnic Institute and State University, Blacksburg, VA, 24060, USA \\ Jaeheon $\operatorname{Sim}^{\dagger}$ and Wei Shyy \\ University of Michigan, Ann Arbor, MI, 48109, USA
}

\begin{abstract}
Computational simulations of multiphase flow are challenging because many practical applications require adequate resolution of not only interfacial physics associated with moving boundaries with possible topological changes, but also around three-dimensional, irregular solid geometries. In this paper, we focus on the simulations of fluid/fluid dynamics around complex geometries, based on an Eulerian-Lagrangian framework. The approach uses two independent but related grid layouts to track the interfacial and solid boundary conditions, and is capable of capturing interfacial as well as multiphase dynamics. In particular, the stationary Cartesian grid with time dependent, local adaptive refinement is utilized to handle the computation of the transport equations, while the interface shape and movement are treated by marker-based triangulated surface meshes which freely move and interact with the Cartesian grid. The markers are also used to identify the location of solid boundaries and enforce the no-slip condition there. Issues related to the contact line treatment, topological changes of multiphase fronts during merger or breakup of objects, and necessary data structures and solution techniques are also highlighted. Selected test cases including spacecraft fuel tank flow management, and movement and rupture of interfaces associated with liquid plug flow are presented.
\end{abstract}

\section{Introduction}

$\mathrm{F}$ LOWS involving interactions between liquids and gases can be observed in a wide range of engineering applications. When capillary effects become significant, such flows are referred to as interfacial flows, in which one needs to account for the multiphase front, or the interface, separating the liquid and the gas state as the interplay between the interface and the flow dynamics determines the outcome of the application mechanics.

Numerical simulations of interfacial flows are required to resolve the location of the interface to apply the conditions arising from surface tension forces and distinctive material properties of the constituents. As reviewed by multiple authors ${ }^{1-6}$, there exist numerous methods for tracking the location and the shape of the interface as well as for applying proper treatments around the interface. The computational techniques for treating moving interfaces are typically categorized into three separate groups:

- Lagrangian methods ${ }^{7,8}$ that modifies the grid to match the interface location,

- Eulerian methods ${ }^{4,9-13}$ that extract the interface location with the help of a scalar function on a stationary grid,

- Eulerian-Lagrangian methods ${ }^{5,14-18}$ that utilize a separate set of grid representing the interface on a stationary grid. Grid that represents the front can move freely based on the solution obtained on the stationary grid.

Once the location is known, various methods are usually employed for establishing the interfacial conditions, which impose the discontinuous pressure and viscous stresses across the interface as a result of the surface tension forces. According to multiple authors ${ }^{1,2,4,19}$, these methods can be observed in two separate groups:

- Continuous interface methods ${ }^{5,14,15,20,21}$ (CIM) that solves one set of equations by smearing out the flow properties around the interface,

\footnotetext{
*Visiting Assistant Professor, Department of Mechanical Engineering, Member AIAA.

${ }^{\dagger}$ Graduate Student, Department of Aerospace Engineering, Student member AIAA.

* Clarence L. "Kelly" Johnson Collegiate Professor and Chair, Department of Aerospace Engineering, Fellow AIAA.
} 
- Sharp interface methods ${ }^{12,22-27}$ (SIM) that impose the conditions directly on the interface by considering different sets of equations for different phases.

During the last decade, various combinations of the interface tracking methods and interfacial conditions models have been proposed in order to capture the interfacial dynamics. As highlighted by Uzgoren et al. ${ }^{1}$, the difficulties that rise during developing a model for interfacial flows are limited not to the algorithmic challenges, but also to the compromise made between the computational cost and accuracy capturing the interfacial dynamics. The computational cost is mainly determined by solution of the non-linear Navier-Stokes equations on local adaptive meshes, the identification and treatment of marker-based triangularized fluid-fluid interface and solid boundary conditions, and interaction between the Navier-Stokes equations and moving interfaces.

The goal of the present study is to develop a cost-effective method for a unified three-dimensional multiphase flow solver that is capable of capturing the interfacial flow dynamics,

- around complex geometries,

- with wetting surface as part of a computational boundary or the irregularly shaped solid geometry,

- with multiple multiphase fronts that define existence of multiple constituents in various phases,

- with topological changes, i.e. possibilities of a merger or a break-up

In order to achieve the goals listed above, the present study utilizes a marker based interface tracking algorithm, which is an Eulerian-Lagrangian method. For interfacial flow conditions at the multiphase fronts, a continuous interface method (CIM) is adopted to allow utilizing a single set of equations. In order to maintain the computational cost at a feasible level, the stationary grid is implemented as a Cartesian grid which employs local adaptation ${ }^{28}$ to fully resolve the flow features at locations near the multiphase front. In this combination, the overall method is referred to as Immersed Boundary Method ${ }^{1,5,14,20,21}$.

Practices of fluid flow simulations oftentimes require capabilities of handling complex geometries within the flow field. The marker based data structure which has already been defined for tracking the multiphase fronts can be utilized to apply the prescribed conditions on the embedded boundary. Consequential technique falls into the category of non-boundary conforming techniques, which are relatively easier to generalize, computationally efficient, and can be used along with Cartesian grids. In contrast to the continuous interface method adopted for the multiphase front, the embedded boundary method for solid geometries fall under the sharp interface methods, as no smearing is involved. Yang and Balaras ${ }^{29}$ demonstrated its capabilities via validation studies including the laminar flow induced by oscillating cylinder, a flow over a cylinder and turbulent flow (modeled by LES) over a traveling wavy wall. A similar approach is utilized for the numerical simulations of flapping wings and insect flight ${ }^{30}$.

When moving multiphase front comes in the vicinity of a wetting surface, defined at a computational boundary or at a complex solid interface, there is a possibility of a tri-junction location formation where all phases (solid, liquid, and gas) meet. The corresponding forces at this location is not only determined by the surface tension between the liquid and gas phases, but also interactions due to solid-liquid, solid-gas phase as well as the adhesive forces keeping the contact line in touch with the solid surface. In static equilibrium, the angle between the solid surface and the multiphase front is described by the Young's equation. The major difficulty in numerical simulations involving a contact line is that the contact line is known to slip on the solid surface, which is usually defined by a no-slip condition. One way to incorporate Young's equation into the numerical simulations of droplets/bubbles on solid surface is to impose the static angle determined directly at the tri-junction location to produce the quasiequilibrium representation of the interface shape ${ }^{21}$. One of the drawbacks of such a treatment is that the modification at the contact line may result in a localized high curvature region on the fluid interface in the vicinity of the contact line. Especially considering the balance between the viscous and surface tension forces in that region, there is a possibility that this high curvature region can create instability for simulations with large Laplace numbers. Instead of imposing the contact angle, another possibility is to let the interface evolve into the equilibrium condition by modeling the force

The formation of a contact line brings another challenge in terms representing the topology of the multiphase front. The markers representing the multiphase front are tracked with the help of connectivity information corresponding to a triangulated surface. This connectivity information allows us to compute geometric information, such as volume and curvature. Oftentimes, the algorithmic difficulty of finding the intersection between the interfaces brings additional computational cost, especially in three dimensional computations. This computational complexity can be reduced significantly by employing an indicator function, which varies from zero to one smoothly across the interfaces ${ }^{18,31}$, to identify the elements intersecting. Besides such a benefit, another benefit of using the indicator function is to allow a single set equation formulation of the continuous interface methods by treating the fluid properties such as density and viscosity across an interface over a finite thickness.

In recent years, interface tracking via triangulated elements have improved its most obvious drawback of algorithmic complexity for the problems in which topological changes occur. This is achieved by means of level- 
contour construction algorithm without the need of the connectivity information ${ }^{32}$. On the other hand, keeping the connectivity information helps to reduce the computational effort as the reconstruction algorithm can be applied locally where it is required ${ }^{18}$.

We highlight the capabilities of the techniques presented in our recent papers ${ }^{1,14,15,18}$ by exploring the following flow problems:

- Attaining equilibrium in three-dimensional capillary tubes to assess the validation of contact line treatment

- Elongated liquid plug experiencing a break-up as a practical application of the liquid drug delivery into the lungs

- Dynamics of the draining process of liquid fuel contained in a spacecraft fuel tank subjected to acceleration in axial and radial directions.

- Sloshing motion of the liquid fuel contained in a spacecraft fuel tank subjected to a sudden reduction in acceleration.

\section{Numerical Methods}

The marker based method employs Eulerian and Lagrangian variables in order to perform the interfacial flow computations. Eulerian quantities are solved on the stationary background grid, whereas Lagrangian quantities arise due to the marker points defined on the interface which can move freely. A single fluid formulation for all fluid phases is made possible by smearing the properties across the interface. Incompressible Navier-Stokes equations for mass and momentum conservation are given in Eqs. (1) and (2) respectively, which accounts for the interfacial dynamics. The source term in the momentum equation, $F_{f}$, represents the conditions of interfaces due to surface tension effects of fluid interfaces, whereas additional source term, $F_{s}$, represents the forcing function to establish noslip condition on solid interfaces.

$$
\begin{gathered}
\nabla \cdot u=0 \\
\frac{\partial \rho u}{\partial t}+\nabla \cdot(\rho u u)=-\nabla p+\nabla \cdot\left(\mu \nabla u+\mu \nabla^{T} u\right)+F_{s}+F_{f}+\rho g
\end{gathered}
$$

In general, the pressure and viscous stresses show discontinuities across a fluid interface related to the surface tension force and fluid property jumps. Equation (3) relates the jump in flow properties (pressure, p, and normal stress components, $n \cdot \tau \cdot n$ ) with the surface tension force, $\sigma \kappa$.

$$
\left(p_{2}-p_{1}\right)-n \cdot\left(\tau_{2}-\tau_{1}\right) \cdot n=\sigma \kappa
$$

On the other hand, solid interfaces match no-slip wall condition with a prescribed velocity field defining the motion of the solid boundaries via the force field created around the solid phases.

Equations (1) and (2) are solved adopting a projection method using staggered grid finite volume formulation as described in prior studies. ${ }^{33,34}$ The pressure and fluid properties are stored at the cell center and the face-normal velocity is stored on Cartesian cell faces. The flow computation follows the following sequence of steps:

\section{Step 1: Predictor-step}

Solve the momentum equation for an intermediate velocity field $U^{*}$ using Eq. (4) where all the known values such as surface tension source, forcing function for solid geometries, gravitation, convection and old time-step viscous term due to Crank-Nicholson method are lumped into $S^{n}$. The term, $a_{v}$, corresponds to the other half of Crank-Nicholson method. Temporal discretization of the convection term uses $2^{\text {nd }}$ order Runge-Kutta integration. The pressure term is approximated using the old time pressure field. Subsequently, remove the effect of pressure term by shifting the velocity field back to obtain another intermediate velocity field $U^{* *}$ using Eq. (5).

$$
\begin{aligned}
& \left(\Delta V \frac{\rho}{\Delta t}-a_{v}\right) U^{*}=-\int_{d A} \nabla P^{n} \cdot d A+f_{v i s c}^{*}+S^{n} \\
& U^{* *}=U^{*}+\frac{\Delta t \nabla P^{n}}{\rho^{n+1}}
\end{aligned}
$$




\section{Step 2: Corrector-step}

Correct the predicted velocity field $\left(U^{* *}\right)$ using Eq. (6). The pressure field for this correction is computed by enforcing the velocity-divergence condition and solving the Poisson equation (Eq. (7)). The divergence of the new velocity field $U^{n+1}$ is zero due to incompressible flow.

$$
\begin{aligned}
& U^{n+1}=U^{* *}-\frac{\Delta t \nabla P^{n+1}}{\rho^{n+1}} \\
& \sum_{\text {face }}\left(\frac{\nabla P^{n+1}}{\rho^{n+1}}\right) \cdot n d A=\frac{1}{\Delta t} \sum_{\text {face }} U^{* *} \cdot n d A
\end{aligned}
$$

The components of the numerical algorithm and their interactions are summarized in Fig. 1.

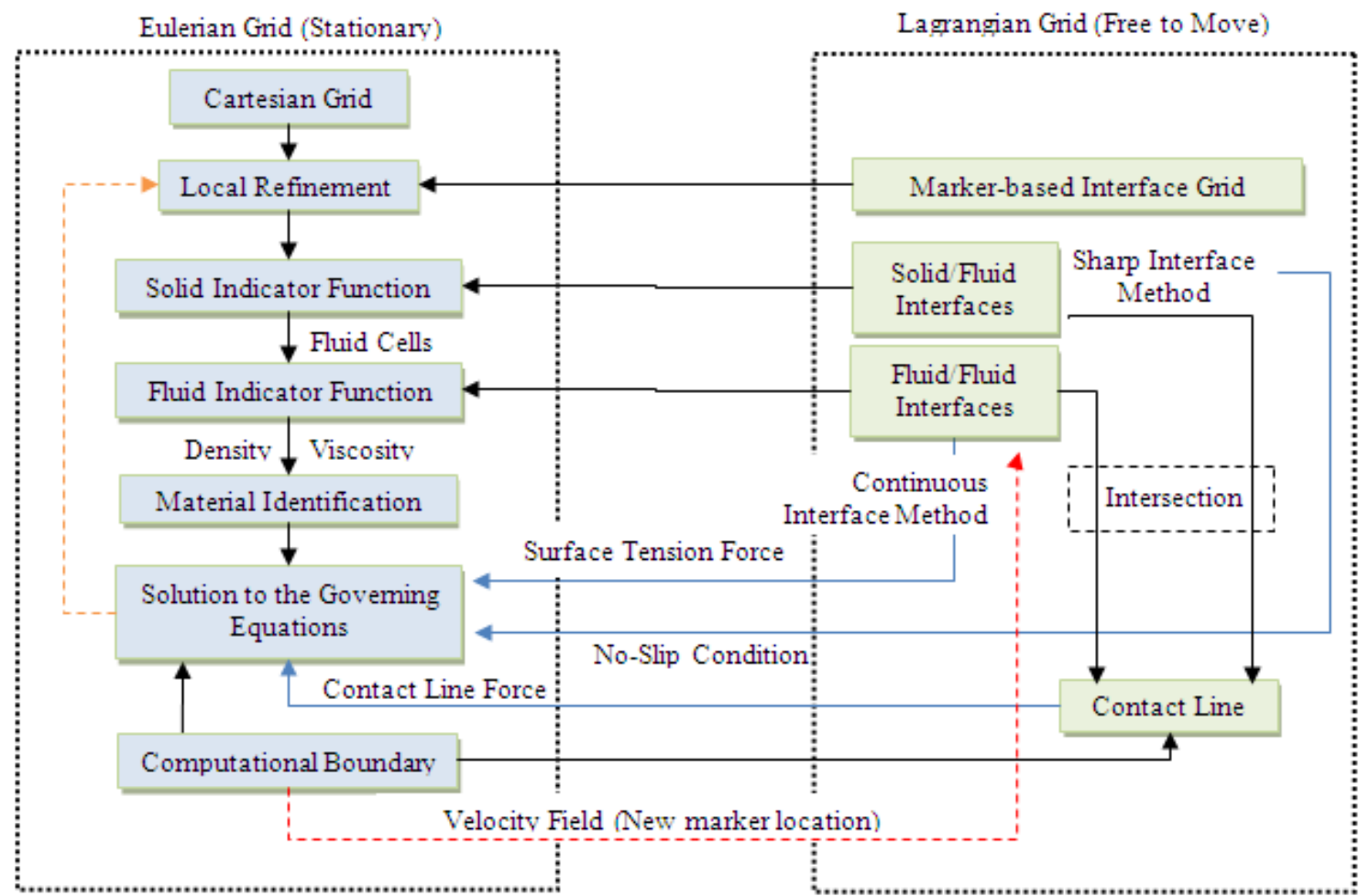

Figure 1. Summary of the numerical method and the interactions between Eulerian and Lagrangian descriptions.

\section{A. Marker Based Interface Tracking}

The interface is represented by marker points, which keep track of the neighboring markers through the maintained connectivity information. The corresponding data structure is established through formation of elements; line-segments in twodimensional computations and triangles in three-dimensional computations, as represented in Fig. 2(a)-(b). Each marker point tracks all the elements that it is connected to while the elements keep

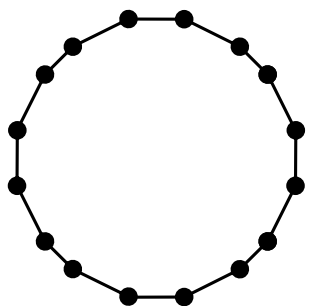

(a)

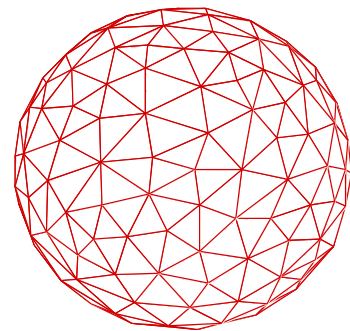

(b)

Figure 2. Interface representation by marker points. (a) Line segments in 2D, (b) Triangular elements in 3D.

American Institute of Aeronautics and Astronautics 
information of the neighboring elements based on the edge that they share. Figure 3 illustrates the connectivity information for two common scenarios; regular 3D element, 3D boundary element. A regular 3D element, or inner element, is when it has three neighboring elements while the boundary element has less than three elements, due to one or more edges being on a boundary. The connectivity information for the boundary edge is represented by the information of the boundary as a negative integer. Absolute value of the boundary index corresponds to the computational boundary for 1 to 6 , reserved for the east, west, north, south, front and back faces of the domain boundary, while the larger numbers representing the elements belonging to a possible solid interface.

The marker locations for the surface grid are computed using the marker velocities as shown in Eq. (10).

$$
\frac{\partial X}{\partial t}=u^{n}(X)
$$

Fluid interfaces use the solution field to compute the marker velocities. Similar to translating the surface forces into the volumetric form, the discrete Dirac delta function is employed for obtaining Lagrangian form of the Eulerian velocity field using Eq. (11). The solid interfaces use the prescribed velocity field to advance the marker points using Eq. (11).

$$
u^{n}(X)=\int_{v} u^{n}(x) \delta(x-X) d v
$$

As the marker points advance to a new position with time, it can lead to an unevenly distributed marker points on the interface. Such a representation would produce large errors in computations. In order to prevent such a scenario, the spacing between marker points is rearranged by addition/deletion whenever two markers come too close or too distant from each other. The criteria is estimated based on the requirements posed by the background grid and. A correction-step to the edge deletion procedure is performed to locally preserve the phase-volumes ${ }^{35}$. Readers may refer to Uzgoren et al. ${ }^{15}$ for further information.

\section{B. Indicator Function}

Cells on the Cartesian grid are represented by a unique material index to identify the constituents separated by interfaces. This brings an algorithmic advantage to identify the interface location as well as to assign proper material properties, i.e. density and viscosity, for flow computations. In order to facilitate a single set of equation formulation of CIM for the whole domain, a smooth variation of discontinuous material properties across interfaces are used. This is achieved with the help of a scalar function, varying from zero to one smoothly. Throughout this document, this function is referred to as the indicator function and denoted by $I$. Once the indicator function is obtained, the fluid properties such as density and viscosity, varying from values between $\varphi_{1}$ and $\varphi_{2}$, are computed using Eq. (12).

$$
\varphi=\varphi_{2}+\left(\varphi_{1}-\varphi_{2}\right) I
$$

First, the material properties are assigned using a simple and efficient method based on the painter's algorithm frequently employed in computer-graphics rendering. Unlike the ray-tracing algorithm, the painter's algorithm does not require expensive computation of three-dimensional line-surface intersection and it is sufficient as the material properties are then corrected with the help of the indicator function, which marks the interface location on the Cartesian grid for the value of 0.5. Such a feature enables handling the geometry related algorithms in a computationally efficient way.

The indicator function is not only utilized for smoothing material properties to facilitate single equation formulation but also brings computational advantages on dealing with interacting complex geometries represented by triangulated surfaces. Obtaining such a function accurately is critical for successful numerically simulating multiphase flow problems. In the literature, two closely related but numerically distinct forms of computations can 
be found. One of these methods adopts the solution of a Poisson equation using the form in Eq. (13), while the others utilize a discrete form of the Heaviside step function (Eq. (14)). Noting that Eq. (13) to be exact, in which indicator function, $I$, yields a Heaviside step function, numerical approximations especially in discrete form of Dirac delta function, $\delta$, and the Heaviside function create differences between these approaches.

$$
\nabla^{2} I=\nabla\left(\int_{A} \delta(x-X) n \cdot d A\right)
$$

In Eq. (13), the location of the interface is represented by the Lagrangian quantity, $X$, while $x$ corresponds to the Eulerian description of the computational domain. Analytical form of the Dirac delta function is only non-zero at $x=X$, where the interface is located. Approximations to the Dirac delta and Heaviside step functions introduce a region that represents the interface over a finite thickness. The properties of these approximations particularly focusing on Dirac delta function have been investigated in prior studies. ${ }^{20,36,37}$

In the present study, the Dirac delta function approximation, that supports the conservation rules dictated by zeroth, first and second moments as described in Peskin, ${ }^{20}$ is employed as the base discrete form using the onedimensional representation given in Eq. (14).

$$
\phi(r)=\left\{\begin{array}{cl}
1-\frac{11}{16}|r|-|r|^{2}-\frac{1}{6}|r|^{3} & 1 \leq|r| \leq 2 \\
1-\frac{1}{2}|r|-|r|^{2}+\frac{1}{2}|r|^{3} & 0 \leq|r| \leq 1 \\
0 & \text { otherwise }
\end{array}\right.
$$

In Eq. (14), $r$ is the closest distance between the cell-center to the interface location, and is normalized by the cell spacing, $h$. Because $\phi(r)$ becomes zero when the distance is larger than two cell width, the smearing region becomes limited to two-cell width on each side of the interface.

One way to extend the one-dimensional representation of the discrete Dirac function to two- and threedimensions is to use the multiplication rule as presented in Eq. (15) $)^{20}$. This approach is attractive due to its low computational cost.

$$
\delta_{h}\left(r_{x}, r_{y}, r_{z}\right)=\frac{1}{h_{x} h_{y} h_{z}} \phi\left(r_{x}\right) \cdot \phi\left(r_{y}\right) \cdot \phi\left(r_{z}\right)
$$

On the other hand, when the information of minimum distance is readily available as in the case of level-set methods, it is possible to utilize the distance function with the one-dimensional form of discrete Dirac function, $\phi$, directly as shown in Eq. (16).

$$
\delta_{h}\left(r_{x}, r_{y}, r_{z}\right)=\frac{1}{\sqrt{h_{x}^{2}+h_{y}^{2}+h_{z}^{2}}} \phi\left(\sqrt{r_{x}^{2}+r_{y}^{2}+r_{z}^{2}}\right)
$$

The indicator function can be obtained via solving Eq. (13) on the Cartesian grid, where it is defined. The discrete form is presented in Eq. (17) for a cell, denoted by $P$.

$$
\sum_{N=N G B R} \frac{\left(I_{N}-I_{P}\right)}{\Delta} A_{N P}=\sum_{N=N G B R}\left(A_{N P} S_{N P}\right)
$$

The right hand side term, $S_{N P}$, is computed at the cell faces using the Dirac delta function as in Eq. (18) to convert the surface quantity into its volumetric form.

$$
S_{N P}=\sum_{l} \delta_{h}\left(r_{x}, r_{y}, r_{z}\right) n_{l} \cdot A_{l}
$$

In Eq. (18), the summation is performed over the interface elements, denoted with $l$, and its contribution on face $N P$ is computed based on $\delta_{h}\left(r_{x}, r_{y}, r_{z}\right)$. The arguments of this function are based on the center of the surface element, $l$, and the center of the face, $N P$. However, in practice computing $S_{N P}$ directly at the faces can create oscillations due to a large gradient in the source term. One remedy to this problem is to apply smoothing via computing $S_{N P}$ at the cell centers and computing face values by linear-interpolation. 
Considering discrete Delta function's support, the computations are only performed over the two-cell width region on each side of the interface to reduce the computational cost of the Poisson equation. Figure 4 illustrates this region for the computation. This region is obtained by determining cells around each surface node with two-cell width radius. The boundary conditions away from the interface are set to yield the desired variation, i.e. from zero to one. In the present study, we choose the convention as the normal direction points towards the indicator function to be one. This approach is computationally effective when the boundary conditions are well defined, i.e. away from the interface location once right hand side term is calculated during computations of surface tension forces to reduce its cost.

On dealing with contact line problems, in which the interface is on a surface, the above approach requires boundary conditions in the vicinity of the interface. Because the variation of the

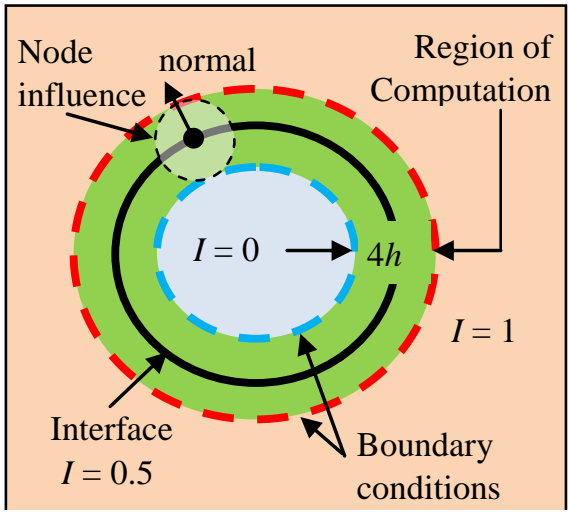

Figure 4. Region of computation and boundary conditions.

indicator function at this region depends on the normal direction, it makes difficult to utilize an appropriate boundary condition. One possible condition is to assume zero variation in the indicator value at the normal direction to the boundary. However, this condition leads to an interface representation that makes $90^{\circ}$ to the domain boundary, which can result in a different interface shape on the Cartesian than the actual interface at angles away from $90^{\circ}$. Using linear extrapolation can also cause incorrect interface line $(I=0.5)$ around this region. Figure 5 illustrates such a scenario.

This issue can be handled using the alternate way of computing the indicator function, which utilizes the shortest distance value between the cell-center to the interface location by the integrating the one-dimensional form of discrete Dirac function as a result of Eq. (19).

Utilization of Eq. (19) requires the determination of the shortest distance, $r$, which can easily increase the computational time when performed on a large surface grid. In order to avoid such a difficulty, similar analogy to what has been described in Fig. 5 is adopted. Specifically, during the isolation of the computational region indicated in Fig. 5, each element computes its distance from its geometric center to the cell-centers which are in their zone of influence and compares it to the distance value contained in that particular cell to find the minimum distance from the cell to the overall interface. In order to overcome the difficulties caused by sharp corners, the distance values are interpolated to face-centers from the cell-centers. Furthermore, this algorithm is coupled with identification of material tags on the Cartesian grid to save

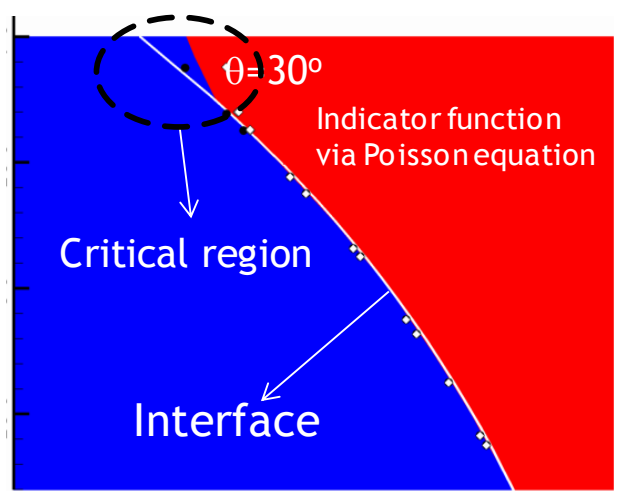

Figure 5. Indicator function constructed solving the Poisson equation misrepresents interface location at angles less than $30^{\circ}$. computational time.

$$
I(r)=\frac{1}{8}\left\{\begin{array}{lc}
0 & r<-2 \\
\frac{23}{4}-2 \pi+5 r+r^{2}-\left(\frac{r}{2}+\frac{3}{4}\right) \sqrt{-4 r^{2}-12 r-7}-\frac{1}{2} \sin ^{-1}\left(-\frac{2 r+3}{\sqrt{2}}\right) & -2<r \leq-1 \\
\frac{15}{4}-2 \pi+3 r+r^{2}+\left(\frac{r}{2}+\frac{1}{4}\right) \sqrt{-4 r^{2}-4 r+1}-\frac{1}{2} \sin ^{-1}\left(-\frac{2 r+1}{\sqrt{2}}\right) & -1<r \leq 0 \\
\frac{17}{4}+2 \pi+3 r-r^{2}+\left(\frac{r}{2}-\frac{1}{4}\right) \sqrt{-4 r^{2}+4 r+1}-\frac{1}{2} \sin ^{-1}\left(-\frac{2 r-1}{\sqrt{2}}\right) & 0<r \leq 1 \\
\frac{9}{4}+2 \pi+5 r-r^{2}-\left(\frac{r}{2}-\frac{3}{4}\right) \sqrt{-4 r^{2}+12 r-7}-\frac{1}{2} \sin ^{-1}\left(-\frac{2 r-3}{\sqrt{2}}\right) & 1<r \leq 2 \\
1 & r>2
\end{array}\right.
$$




\section{Adaptive Grid}

Multiphase flow problems involve multiple length scales. In order to effectively resolve the flow features in such cases, the present study considers adaptive grid refinement employed on Cartesian grids. The approach is based on isotropic refinement which splits the cells into four and eight equal sibling cells in two- and three-dimensions, respectively. The grid is represented using an unstructured data that connects cells through cell faces. The details of the algorithm can be found Singh and Shyy. ${ }^{35,38}$ Adaptation is performed based on the interface location and the flow solution quality.

The geometry-based adaptation near interfaces has different characteristics based on what interfaces represent. The fluid interfaces, for which discontinuous flow properties smeared across two layers of cells, perform refinement to achieve full resolution in their vicinity in order to capture interfacial dynamics accurately. This fully resolved region around a fluid interface is further extended to six layers of cells to handle both discrete Dirac function as well as a possibility for large deformation of interface. Figure 6 illustrates the process of geometry based adaptation starting from a uniform base grid and refining up to four levels. On the other hand, solid interfaces, which do not employ discrete Dirac function, triggers full refinement on vicinity including two-cell layers from the interface.

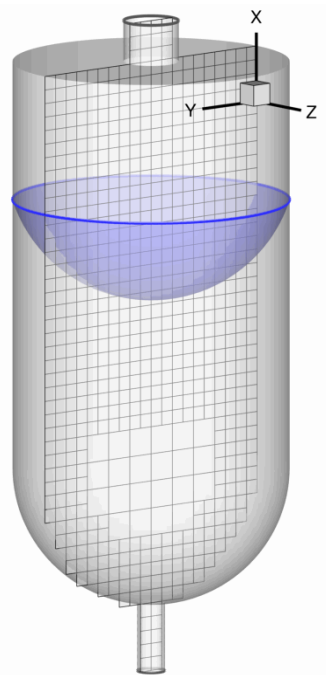

(a) Level $=1$

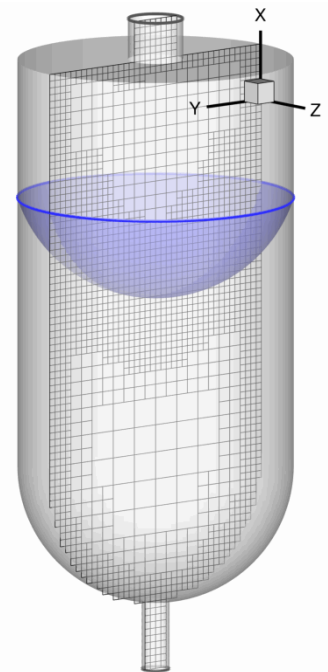

(b) Level $=2$

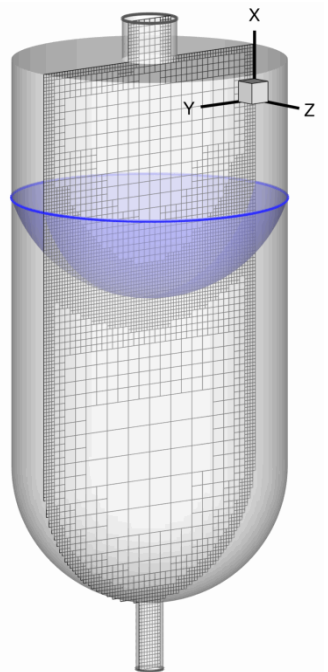

(c) Level $=3$

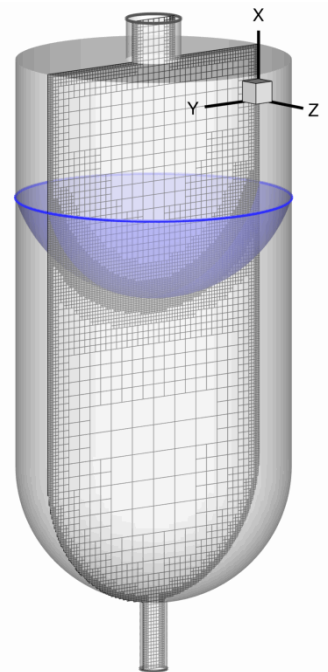

(d) Level $=4$

Figure 6. Snapshots of geometry-based grid adaptation for fluid interfaces.

Cells, those are not already fully refined, are adapted based on solution (of the flow field). The present implementation uses a curl based adaptation criterion ${ }^{39}$ that computes a parameter $\xi$ for each cell as shown in Eq. (20). The length scale $l$ is estimated as the cubic root of cell-volume. The decision to refine or coarsen a cell is made by comparing $\xi_{\text {cell }}$ to the standard deviation (Eq. (21)) using the criteria in Eqs. (22) and (23).

$$
\begin{gathered}
\left.\xi_{\text {cell }}|\sim| \nabla \otimes U\right|^{I} \\
\sigma^{\prime}=\frac{1}{N_{\text {cell }} \sum_{i} \xi_{i}^{2}} \\
\xi_{\text {cell }}>\sigma^{\prime} \rightarrow \text { Refine cell } \\
\xi_{\text {cell }}>0.1 \sigma^{\prime} \rightarrow \text { coarsen the cell }
\end{gathered}
$$

During the adaptation procedure, the Cartesian cell center values such as pressure, temperature and face normal velocities need to be reconstructed for the newly created cells and faces. Flow variable reconstruction during cell and face coarsening is performed simply by averaging of the corresponding cell-centered or face-centered values. Because the adaptation algorithm is triggered during the predictor step, just before solving the pressure Poisson equation, the reconstruction algorithm is not required to satisfy the divergence free velocity condition for $U^{* *}$. 


\section{Fluid Interface Treatment}

\section{Surface Tension}

When interface separating fluid phases, the source term arises from the surface tension $(\sigma)$ and the curvature $(\kappa)$ as shown in Eq. (24).

$$
F_{S}=\int_{S} \sigma \kappa n \delta(x-X) d S
$$

The surface force is computed using the Lagrangian marker points, $X$, and is translated into an Eulerian quantity, $x$, via the approximate discrete Dirac delta function, $\delta(x-X)$. After these equations are solved, approximate Dirac delta function is also used for obtaining the marker velocity field to move marker points for obtaining the new geometric surface representation.

The surface tension force is computed on the interface triangles. The surface tension force on a discretized interface element (curves in 2D and triangles in 3D) can be evaluated in several ways: computation with Eq. (25) where unit normal vector and curvature can be computed using curve fitting for two-dimensional interfaces ${ }^{21,22,40}$ and surface fitting for three-dimensional interfaces $;^{41}$ computation using a line integral form shown in Eq. (26) and fitting curves/surfaces to obtain normal and tangent vectors. ${ }^{5,42}$

$$
\begin{gathered}
\delta f=\int_{\delta A} \sigma \kappa n d A \\
\delta f=\int_{\delta A} \sigma(n \times \nabla) \times n d A=\int_{S} \sigma t \times n d s
\end{gathered}
$$

There are two important observations to be made here: the net surface tension force on a closed surface should be zero (conservation); curvature computation using interpolation based methods are numerically sensitive and often requires some form of data smoothing. ${ }^{21,40,41,43}$ The use of Eq. (25) does not enforce conservation whereas the line-integral form, Eq. (26), does not require explicit curvature computation and maintains the conservation.

The approach developed by $\operatorname{Singh}^{35}$ uses the line integral form and computes the local normal and tangent vectors along the triangle edges using the simple approach of Al-Rawahi ${ }^{42,43}$ shown in Eq. (27) following Fig. 7. If required, the curvature can be computed using Eq. (28). Such a simple technique is seen to produce sufficient accuracy demonstrated by Fig. 7, comparing curvature of a unit circle using present method and a cubic-spline interpolation. The overall accuracy of this approach to compute surface tension force and its modeling have already been demonstrated for boiling flows ${ }^{44}$ and for

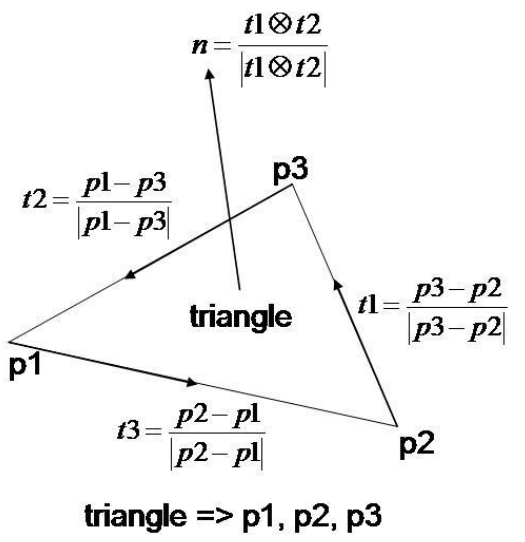

Figure 7. Computation of the unit normal and tangent vectors on interface triangles. dendritic solidification ${ }^{43}$.

$$
\begin{gathered}
\delta f=\sum_{e d g e=123} \sigma(t \otimes n)_{e d g e} \Delta s \\
\kappa=\frac{\delta f \cdot n}{\sigma \Delta A}
\end{gathered}
$$

\section{Van der Waals Forces}

Additional physical mechanisms at the interface can be computed using the same methodology described for the surface tension forces. One of these forces considered in this study is the van der Waals attraction forces, which 
describes the interaction between film surfaces due to molecular force attraction using a potential. The potential describing the attraction is defined based on the minimum distance between the interfaces:

$$
\phi(r)=\frac{A^{\prime}}{r^{3}}
$$

In the above equation, $A^{\prime}$, is the Hamaker constant describing the strength of the potential based on the materials involved. The force field due to Waals potential can be estimated as follows:

$$
\begin{gathered}
\boldsymbol{F}_{\boldsymbol{w}}=\boldsymbol{\nabla} \phi=\frac{\partial \phi(r)}{\partial x} i+\frac{\partial \phi(r)}{\partial y} j+\frac{\partial \phi(r)}{\partial z} k \\
=\frac{\partial \phi}{\partial r} \frac{\partial r}{\partial x} i+\frac{\partial \phi}{\partial r} \frac{\partial r}{\partial y} j+\frac{\partial \phi}{\partial r} \frac{\partial r}{\partial z} k=\frac{\partial \phi}{\partial r} \boldsymbol{r} \\
\boldsymbol{F}_{\boldsymbol{w}}=-\frac{3 A^{\prime}}{r^{4}}\left(r_{x} i+r_{y} j+r_{z} k\right)
\end{gathered}
$$

The modified incompressible Navier-Stokes equations then incorporate this force as a source term in the momentum equation, similar to the surface tension force treatment.

When $r$ becomes smaller, the attraction forces becomes significant leading to a modification to the interface shape. This change can be responded by the surface tension forces due to newly formed curvature effects. The interface shape can be recovered if curvature effects are dominant than the attraction forces. If the attraction forces are larger, the interfaces reaches a critical value of $r$, for which the surface tension forces can no longer compete with the attraction forces. At this stage, the film thickness becomes smaller and smaller, eventually leading to a topological change. In order to identify this critical film thickness, the ratio between the non-dimensionalized surface tension and van der Waals attraction forces is used. The characteristic length is chosen as the film thickness to yield Eq. (33).

$$
\frac{\rho_{1}}{\mu_{1}^{2}}\left(\sigma h_{o}-\frac{A^{\prime}}{h_{o}}\right) \rightarrow h_{o}^{2} \sim \frac{A^{\prime}}{\sigma}
$$

\section{E. Solid Interface Treatment}

Solid interfaces are modeled using a sharp interface method that imposes the prescribed conditions on an arbitrary interface by reconstructing a force field around a solid phase. Using Eq. (2), the source term due to solid interfaces, $\mathrm{F}_{\mathrm{s}}$, can be estimated at its non-zero locations with the help of the prescribed velocity at the interface, $u_{\text {int }}$, as in Eq. (34).

$$
F_{s}=\rho \frac{\partial u_{i n t}}{\partial t}+\nabla \cdot(\rho u u)_{i n t}-\nabla p-\nabla \cdot\left(\mu \nabla u+\mu \nabla^{T} u\right)_{i n t}-F_{f}-\rho g
$$

Following the prior naming by Yang and Balaras ${ }^{29}$, these nonzero locations are referred to as forcing faces as the present study considers a staggered variable arrangement, in which the velocity components are defined at the face-centers and the forcing field is formed using the face-centers of the cells surrounding the solid interfaces.

Recalling one of the merits of Harlow-Welch type staggered grid, there is no need for the artificial pressure boundary conditions in the continuity equation ${ }^{45,46}$. At locations, where the boundary conditions are defined exactly on the face-centers, i.e. computational boundaries, the procedure of solving the pressure Poisson equation is presented in Eq. (35) with the guidance of Fig. 8.

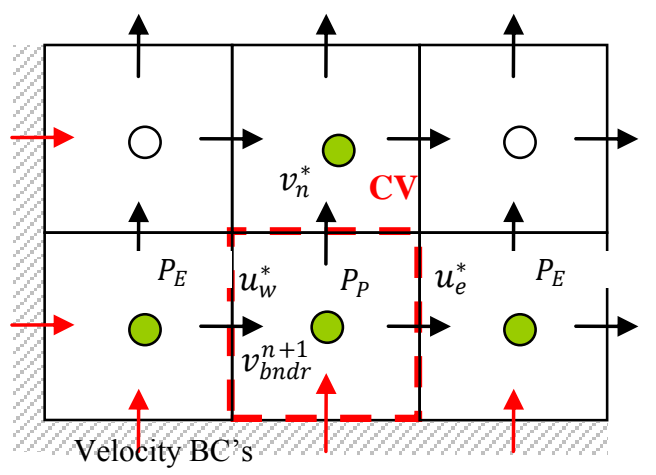

Figure 8. Pressure control volume at the computational boundaries. 


$$
\frac{P_{E}-P_{P}}{\Delta x} A_{e}+\frac{P_{W}-P_{P}}{\Delta x} A_{w}+\frac{P_{N}-P_{P}}{\Delta y} A_{N} A_{S}=u_{e}^{*} A_{e}-u_{w}^{*} A_{w}+v_{n}^{*} A_{n}-v_{b n d r}^{n+1} A_{s}
$$

The last term of the right hand side term in Eq. (30), is the total mass flux at the boundary face and the corresponding pressure gradient term on the left hand side term is not needed as mass flux is known at the next time step. Similar procedure is adopted for the forcing faces. Hence the identification procedure considers the pressure Poisson equation on the fluid side and marks the faces as forcing if the material on the other side indicates a solid material flag. This study utilizes negative values for solid phase and positive values for the fluid phases. As a result, any cell that has a negative index value is removed from the solution procedure of the Poisson equation. These faces form the first set of the forcing faces.

Additional faces are also marked as forcing faces due to the nature of the prediction step of the projection method. When we consider the momentum equation, the viscous and advection terms require another set of faces that would yield a correct gradient at the boundary layer. In this study, this set of faces is chosen on the solid side as shown in Fig. 9. It should also be noted that, in some problems dealing with thin or zero-thickness solid interfaces, these faces can also be chosen from the fluid side ${ }^{29,30}$. However, this approach would make the construction of the interpolation scheme difficult especially at the inner corner locations, where less than sufficient fluid faces exist.

Once the forcing faces are set, the forcing terms on these faces are computed using a linear interpolation scheme between the prescribed velocity field on the interface, and the predicted velocity field at the fluid side. The first point on the interpolation scheme, the closest location on the interface from the forcing face, is found by comparing the distance normalized by the grid spacing for the elements in the vicinity of the forcing face. Once determined, interpolation weights based on inverse distance is computed using Eq. (36) as the shortest possible distance does not necessarily need to be from the markers (Fig. 10). Then the prescribed condition on the interface can be obtained for any function, $\phi$, using Eq. (37).

$$
\begin{gathered}
w_{i j}=\frac{1 / \Delta_{i j}}{\sum_{i=1,3}\left(1 / \Delta_{i j}\right)} \\
\phi_{j}=\phi_{1} w_{1 j}+\phi_{2} w_{2 j}+\phi_{3} w_{3 j}
\end{gathered}
$$

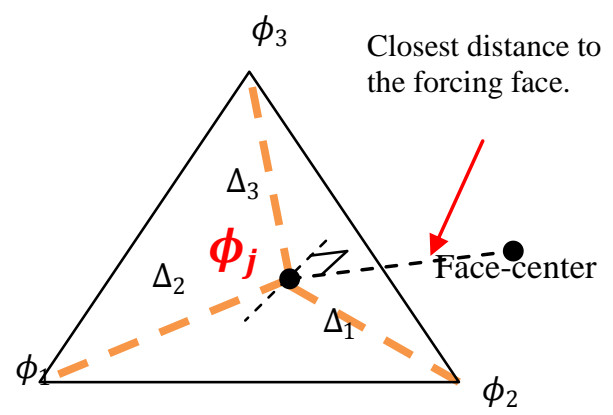

Figure 10. The closest interface element to a forcing face.

geometrically represented in the shape of a triangle in 2D, and a tetrahedron in 3D as illustrated in Fig. 11. fluid faces, identification of which is one of the most critical parts of the algorithm. The accuracy of the interpolation scheme improves when those fluid faces are selected as close as possible to the forcing face while avoiding an illconditioned scheme, which can happen when some of the interpolation points are aligned together. To satisfy the requirements for shortest distance and avoid ill-conditioned scheme between the face-centers included in the scheme, a short list of liquid faces is formed using the neighboring cells. This list is sorted using a merge-sort algorithm based on the distance values. The various combinations of faces are checked for their cross-product to verify their alignments starting from the best qualified distance values. This procedure results in an interpolation scheme, which can be

The remaining points in the interpolation scheme are the 
The interpolation procedure is performed assuming a linear variation of any variable, $\phi$. Equations (38) and (39) are the formulation of the procedure in $2 \mathrm{D}$.

$$
\begin{gathered}
\phi=b_{1}+b_{2} x+b_{3} y \\
{\left[\begin{array}{l}
b_{1} \\
b_{2} \\
b_{3}
\end{array}\right]=\left[\begin{array}{lll}
1 & x_{1} & y_{1} \\
1 & x_{2} & y_{2} \\
1 & x_{3} & y_{3}
\end{array}\right]^{-1}\left[\begin{array}{l}
\phi_{1} \\
\phi_{2} \\
\phi_{3}
\end{array}\right]}
\end{gathered}
$$

In Eqs. (33) and (34), $x_{i}$ and $y_{i}$ represents the corners of the triangle presented in Fig. 11. For stationary objects, the coefficients can be obtained once and then be used for reconstructing the velocity field at each time step. On the other hand, the system has to be solved at every time step for moving boundaries. 3D computations are achieved in a similar manner by adding an additional point to obtain the coefficient of the $z$-coordinate, $b_{4}$.

The above interpolation scheme is utilized in the estimation of the forcing term, $F_{s}$, in Eq. (2). Because this term is computed at the prediction step of the projection method, it is not computed explicitly which is the case for surface tension forces, $F_{f}$, for the fluid interfaces. Instead, it is reflected in the predicted velocity field using the interpolation scheme discussed in the prior sections. These forcing velocity terms are utilized in the prediction step to determine the flux computation in the advection-diffusion equation as well as the mass flux values in the continuity equation as described in Eq. (35).

The corrected velocity field is required to satisfy the divergence free condition both locally and globally. In the staggered grid configuration, having the sum of local control volumes equal to the global control volume, this condition is automatically satisfied when the correct mass flux information is used at the boundary cells that utilize the concept presented in Eq. (35). As one may expect, the constructed forcing velocity field does not impose the conditions required by the continuity equation and hence is not divergence free.
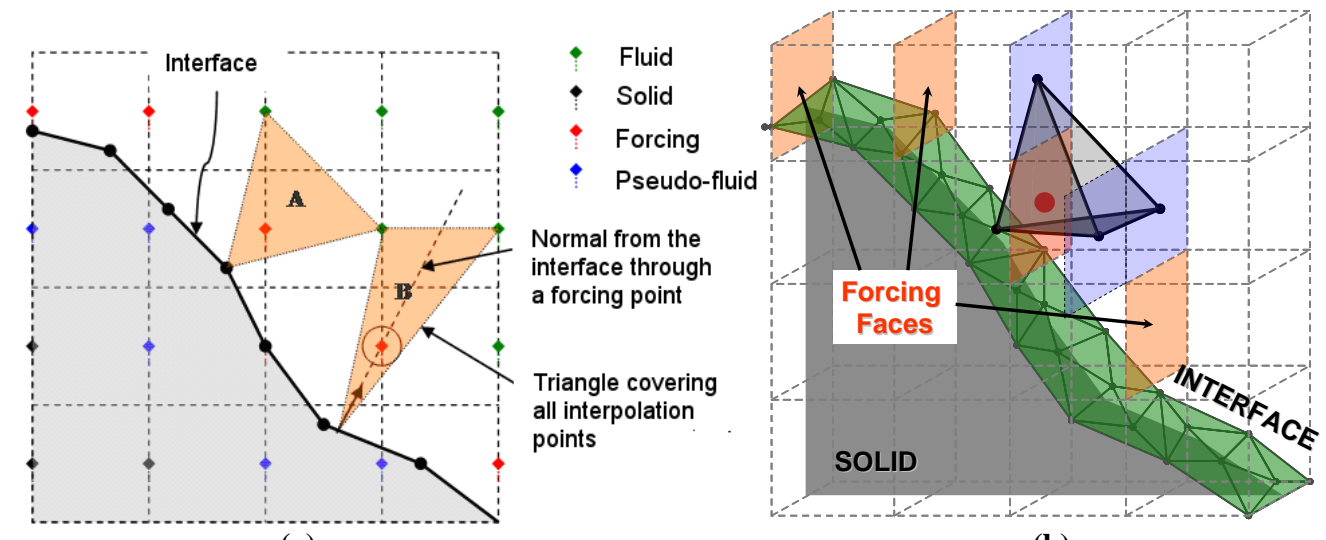

(a)

(b)

Figure 11. Definition of faces around the solid interface for u-velocity (a) in 2D, (b) in 3D.

Figure 12 is an example to illustrate the correction algorithm. The forcing faces that divides solid and fluid creates staircase-like boundary faces for the pressure Poisson equation. Let $u^{*}$ and $v^{*}$ be the forcing velocities defined at the faces between fluid and solid phases. The correct flux to be included in the $\mathrm{x}$ face needs to account for the area that is cut by the interface, $\Delta A_{x}^{\prime}$ instead of the full area of the face, $\Delta A_{x}$. It is a similar case for the $y$-face, that is $\Delta A_{y}^{\prime}$ instead of $\Delta A_{y}$. Hence, assuming the velocity field, $u^{*}$ and $v^{*}$, is divergence free, Eq. (40) should be satisfied, resulting an error, $e$, when the full area for each face is utilized as given in Eq. (41).

$$
u^{*} \Delta A_{x}^{\prime}-v^{*} \Delta A_{y}^{\prime}=0
$$




$$
u^{*} \Delta A_{x}-v^{*} \Delta A_{y}=e
$$

The determination of correct face areas requires finding intersection points between the interface grid and the Cartesian grid, which can be computationally challenging and expensive. Instead, the requirement can be obtained by introducing Eq. (42), a correction term for the forcing face velocities to adjust the fluxes accordingly.

$$
\begin{gathered}
e=\epsilon\left(\Delta A_{x}+\Delta A_{y}\right) \\
\left(u^{*}-\epsilon\right) \Delta A_{x}-\left(v^{*}-\epsilon\right) \Delta A_{y}=0
\end{gathered}
$$

When this correction is applied cell-by-cell, the global conservation is automatically satisfied as a result of their individual sum at the corresponding control volumes. This is illustrated in Fig 13. The condition that needs to be satisfied is the divergence free mass flux at the region enclosed by the irregular interface line, marked with red color. The condition is enforced locally at the black line on the regular Cartesian boundary faces, where the material tag changes sign. In Fig. 13, this corresponds to a layer of cells, colored dark. This also satisfies the global conservation requirement on the other side of the bold Cartesian boundary cells.

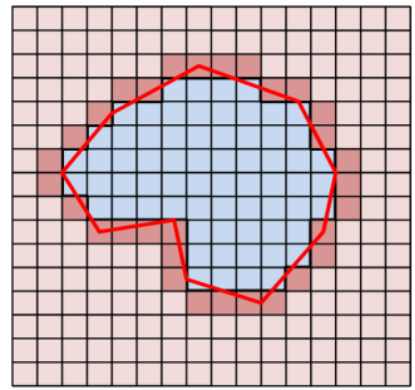

Figure 13. Global conservation with local correction.

Starting from a divergence free velocity field at a given time step, $n$, the forcing function is incorporated in the predicted velocity field, $u^{*}$, on the forcing faces using Eq. (38) leaving the fluid faces at the $u^{n}$ values. This corresponds to the first step in Table 1, which summarizes the algorithm advancing from time step [ $n]$ to time step $[n+1]$. The second step involves the correction step on the first set of forcing faces, which is required only by the Poisson equation. The idea behind the correction is to enforce the global correction based on the way that the cells are cut, so that the continuity equation becomes well-posed. The rest of the terms in the momentum equation are accounted for on solving the advection-diffusion equation to predict the velocity field as appears in third item in Table 1. The fluxes for convection and diffusion equation are computed using the conditions posed by all of the forcing faces and the predicted velocities are updated only at the fluid faces while faces on the solid side are ignored. Similarly, pressure field, defined at the cell centers, are solved at only on the fluid side using the fluxes computed by predicted velocity field for fluid faces and the fluxes at the corrected forcing faces. Hence, the correction is only applied on the fluid faces to satisfy the divergence free condition for the next time step.

Table 1. Overall algorithm for solid boundary treatment

1. Apply forcing terms: $\quad u_{i}^{*}=\left\{\begin{array}{cl}u_{i}^{n} & i \rightarrow \text { Fluid faces } \\ b_{i 1}+b_{i 2} x_{i}+b_{i 3} y_{i}+b_{i 4} z_{i} & i \rightarrow \text { Forcing faces }\end{array}\right.$

2. Apply local correction for global conservation

$$
u_{j}^{n+1}=u_{j}^{*}+\epsilon_{j} \quad j: \text { Forcing faces required only by Poisson equation }
$$

3. Solve for advection diffusion equation:

$$
\begin{gathered}
\left(\Delta V \frac{\rho^{n+1}}{\Delta t}-a_{v}\right) u^{* *}=-\int_{d A} \nabla p^{n} \cdot d A+f_{v i s c}^{* *}-f_{\text {conv }}^{* *}+f_{f}^{n+1}+g \\
u_{i}^{* * *}=u_{i}^{* *}+\frac{\Delta t\left(\nabla p^{n}\right)_{i}}{\rho^{n+1}}
\end{gathered}
$$

4. Solve for continuity equation (pressure Poisson equation)

$$
\sum_{i}\left(\frac{\left(\nabla p^{n+1}\right)_{i}}{\rho^{n+1}}\right) \cdot n d A=\frac{1}{\Delta t} \sum_{i} u_{i}^{* * *} \cdot n d A+\frac{1}{\Delta t} \sum_{j} u_{j}^{n+1} \cdot n d A \quad \begin{aligned}
& i: \text { only fluid faces } \\
& j: \text { only forcing faces }
\end{aligned}
$$

5. Correct velocity field: $\quad u_{i}^{n+1}=u_{i}^{* * *}-\frac{\Delta t\left(\nabla p^{n+1}\right)_{i}}{\rho^{n+1}} \quad i$ : only fluid faces 


\section{F. Contact Line Treatment}

When we consider a fluid-fluid interface intersecting a solid surface, the treatment of the tri-junction locations needs to account for the presence and interactions of all three phases, fluid-fluid-solid, which can be challenging. One of the mostly discussed issues for modeling the tri-junction location, or the contact line, with Navier-Stokes equations is that the imposed no-slip condition for velocity leads to a non-integrable singularity in stress. Among the various models, ones which produce slip condition ${ }^{47,48,49}$ is adopted in this study.

The angle at the contact line, shown in Fig. 14, can be used for representing by the balance of forces resulting from different intermolecular forces between solid, liquid and gas phases. The tangential component of the resulting force, $F_{R}$, is shown in Eq. (44). In static equilibrium, Eq. (44) leads to the well-known Young's equation as given in Eq. (45).

$$
\begin{gathered}
F_{R}=\sigma_{s g}-\sigma_{s l}-\sigma \cos \theta \\
\sigma_{s g}=\sigma_{s l}+\sigma \cos \theta_{0}
\end{gathered}
$$

In Eq. (44), $\sigma_{s g}$ is the surface force due to the interaction of solid and gas, $\sigma_{s l}$ is the surface force due to the interaction

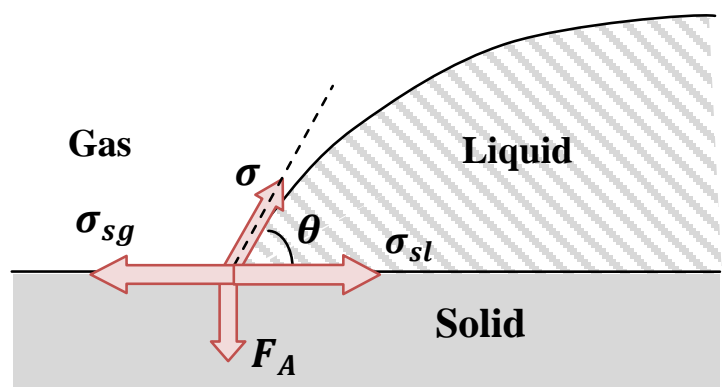

Figure 14: Forces at tri-junction (contact line). $F_{A}$ represents the adhesive forces.

of solid and liquid and $\sigma$ is the surface tension defined for liquid and gas.

In this work, we adopt an approach based on a simplified version of Huang et al. ${ }^{49}$ without considering the effects of the slip velocity on the contact angle. In the present work, flow dynamics moves the contact angle asymptotically towards a prescribed static contact angle. The force at the contact line is obtained by plugging Eq. (45) into Eq. (44), to estimate the force to recover the static contact angle.

$$
F_{R}=\sigma\left(\cos \theta_{0}-\cos \theta\right)
$$

During the computation of the source term due to surface tension, the contact line region contributes to the recovery force described by Eq. (46), instead of the curvature effects given in Eq. (27). The contact angle is enforced on open edges where the element connects to either a computational boundary or a solid interface by modifying Eq. (27) into Eq. (47). In Eq. (47), the angle, $\theta$, defined by the solid surface and the line formed by the mid-point of the edge and the node across. The difference in cosine values between the computed angle, $\theta$, and the prescribed static angle, $\theta_{0}$, is translated into a force acting on the contact line. For a static problem, i.e. capillary tube simulation, this force alone derives the contact line into a given static contact angle from any given initial condition.

$$
\delta f=\sum_{\substack{i=\text { closed } \\
\text { edges }}} \sigma(t \otimes n)_{i} \Delta s+\sum_{\begin{array}{c}
i=\text { open } \\
\text { edges }
\end{array}} \sigma\left(\cos \theta_{0}-\cos \theta\right)_{i} \Delta s
$$

The slip velocity condition on interface markers at the contact line is imposed during the transfer of Eulerian velocity field into the Lagrangian marker points. Instead of imposing the full Dirac function region in Eq. (11), we consider a one-sided averaging via Dirac function. This treatment is represented by Eq. (48).

$$
U(X)=\frac{\sum_{x} u(x) \delta(X-x)}{\sum_{x} \delta(X-x)}
$$

In Eq. (48), the summation of delta function in denominator will yield unity (as a property of delta function) further away from the contact line whereas it is less than unity for markers in the proximity of a solid surface. As a result, casting the velocity field on the contact line yields a slip condition on the solid surface.

\section{G. Intersecting Solid and Fluid Interfaces}

The contact lines can occur not only on the boundaries of the fixed Cartesian grid but also at the solid interfaces that have irregular geometries. Computing the contact line force requires identification of the angle between the fluid and solid interfaces. In order to estimate the contact angle, it is crucial to know which elements of fluid and solid interfaces are in contact. This requirement is imposed by identifying the intersection marker and relating the corresponding fluid and solid markers/elements with each other.

This identification process starts during the computation of the indicator function. The material properties assigned in a particular order, starting with solid interfaces followed by fluid interfaces. This allows us to leave an 
identification number for solid marker on the Cartesian grid cells. As the indicator function computation involves the shortest distance, each cell around a solid interface keeps the index of the closest solid marker point. This information is then used among the fluid interfaces to determine whether they are in the vicinity of a solid boundary.

When there is wetting on a solid interface, the fluid interfacial structure includes open elements, as described earlier in section B. The open elements lack of connectivity information on one of their edges to another element. The markers on this edge is extended or trimmed to fit on the closest solid interface. During this process, the orientation, i.e. normal direction, of the element is maintained.

The identification of open elements in a fluid interface is achieved by utilizing the indicator function which is available on the Cartesian grid. The information of solid indicator function, $I_{s}$, is transferred onto fluid interface markers, $X_{f}$, via Eq. (49) from the Cartesian grid cells, $x$.

$$
I_{s}\left(X_{f}\right)=\int_{v} I_{s}(x) \delta(x-X) d v
$$

When the value of $I_{s}$ at any marker is less than half, which corresponds to the location of the solid interface, it is marked to be trimmed. In addition, elements having markers all to be trimmed are removed from the fluid interface list. For elements having some markers lying inside and outside the solid interfaces are marked as open elements, for which the connectivity information is filled with the corresponding solid marker point, determined by checking index value of the closest solid marker from the underlying Cartesian grid cell.

Once the intersecting solid and fluid elements are known, markers on the open edge are moved on to the solid interface using the distance information as shown in Fig. 15.

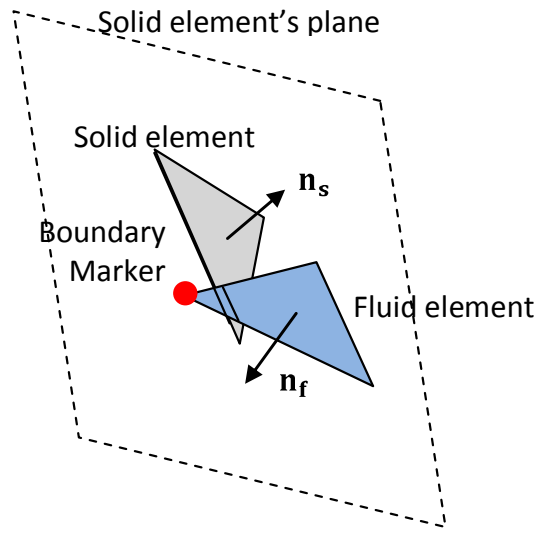

(a)

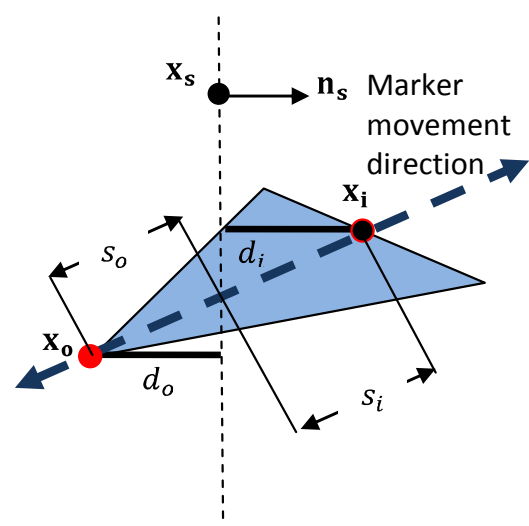

(b)

Figure 15. Intersecting fluid and solid interfaces. (a) intersection between elements (b) Snapping procedure (point of view is tangential direction to the solid element. Marker on the open edge is marked with red.

\section{Computational Assessment}

To highlight the performance of the present approach, case studies have been conducted for (i) Capillary tube simulation at zero gravity with various contact angle and parameters (ii) liquid plug problem, and (iii) 3D simulation of spacecraft fuel tank dynamics including liquid fuel draining at a tilted micro-gravity and liquid sloshing motion under a sudden change of accelerational direction and/or magnitude. These cases are presented in the following.

\section{A. Capillary Tube Simulation}

To validate the ability of the current approach for contact angle and solid interface, the Capillary tube with initially flat interface was simulated at zerogravity condition varying contact angles and non-

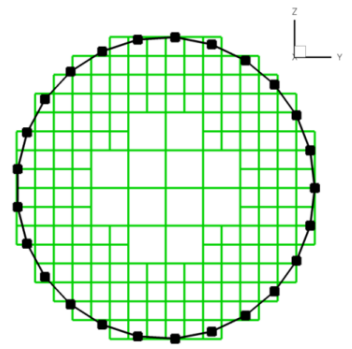

(a)

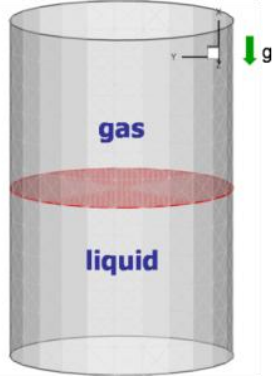

(b)
Figure 16. The computational configuration for 3D Capillary tube simulations. (a) adaptive Cartesian grids and tube wall represented by solid interface, (b) configuration with initial flat interface. 
dimensional parameters. The initial flat interface between gas and liquid is deformed into the curved steady interface shape with the given contact angle by the contact line force applied on the tri-junction point between gas, liquid, and solid wall. The grid convergence and accuracy for various contact angles were first studied in axisymmetric domain. Then, 3D simulations were conducted by representing the circular tube wall using solid interface with triangular element in Cartesian adaptive background grid. These configuration and initial flat interface in a Capillary tube are shown in Fig. 16. The density ratio and viscosity ratio are 1000 and 100 respectively. The Bond number, $B o=$ $\rho g L^{2} / \sigma$, is zero for zero-gravity condition, and it is tested by varying Laplace number, $L a=\rho \sigma L / \mu^{2}$, from $10^{2}$ to $10^{5}$.

The height of liquid column $(\Delta \mathrm{H})$, the difference between wall attachment point and centerline location, is normalized by the radius of tube $(\mathrm{R})$ and chosen for verifying the performance of present contact angle model. Figure 17 shows the comparison between the analytical solution ${ }^{46}$ and the present axisymmetric computation results with various contact angles from $0^{\circ}$ to $180^{\circ}$ at $L a=10^{4}$. Maximum 129 grid points are used along tank radius based on the grid convergence test. The current contact angle model works very well with various contact angles, and shows good consistency with theoretical values especially from $15^{\circ}$ to $165^{\circ}$, where the difference is just around one cell distance.

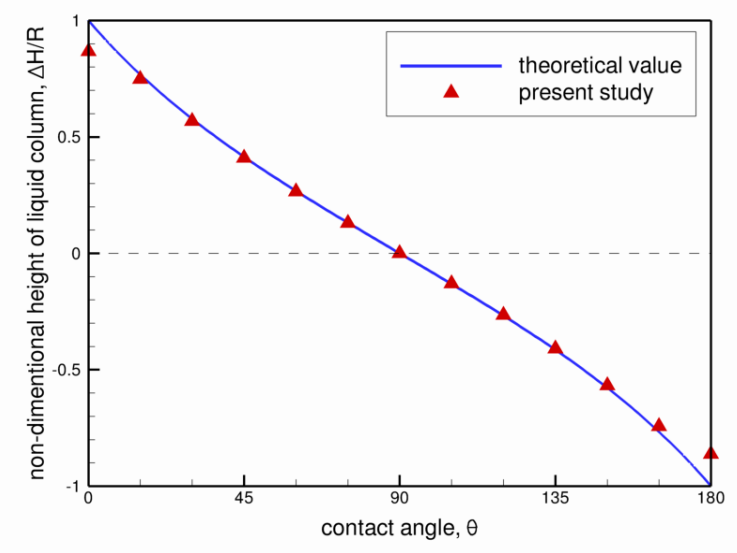

Figure 17. Comparison of non-dimensional height of liquid column between theoretical values and present axisymmetric computation. $L a=10^{4}$, and a maximum of 129 grid points are used along radial direction.

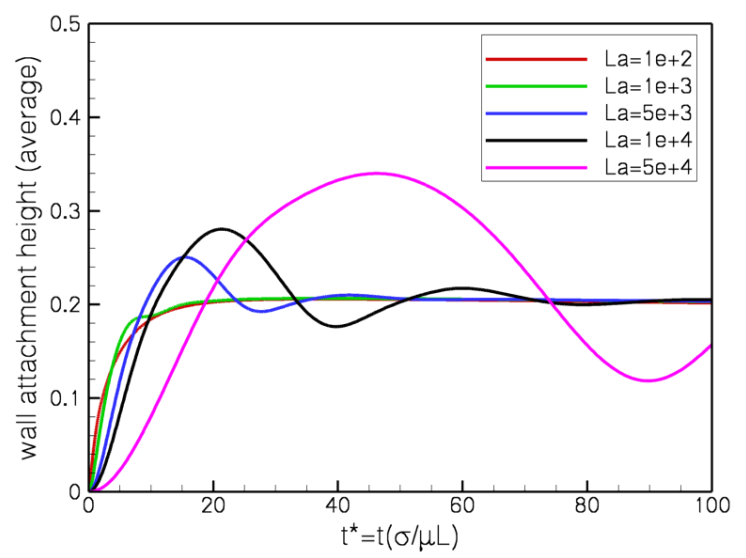

Figure 18. The wall attachment point of 3D Capillary tube with $30^{\circ}$ contact angle. The Laplace number varies from 100 to 50,000 .

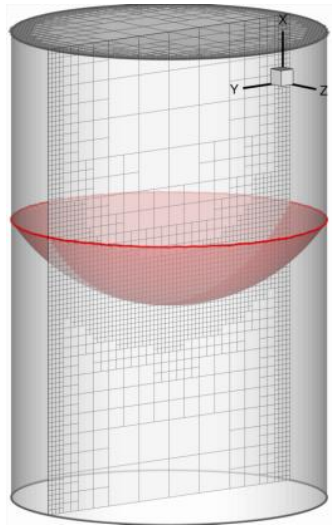

(a) $\theta=30^{\circ}$

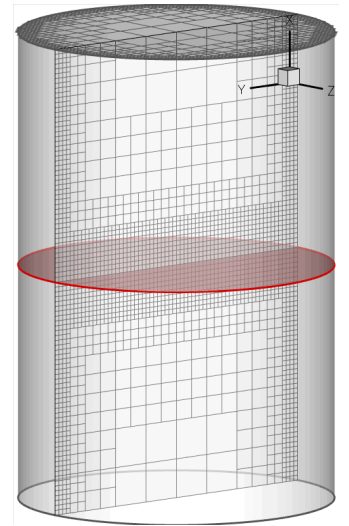

(b) $\boldsymbol{\theta}=90^{\circ}$

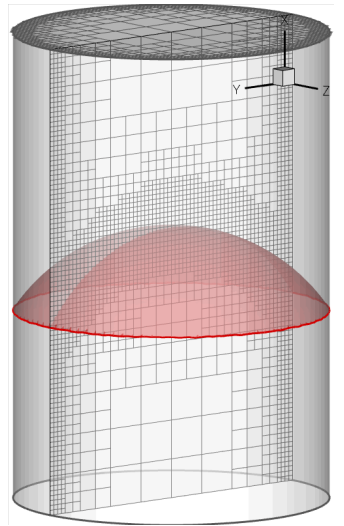

(c) $\theta=150^{\circ}$

Figure 19. The steady interface shape of 3D Capillary tube for different contact angles. (a) $30^{\circ}$, (b) $90^{\circ},(c) 150^{\circ}$ 
The 3D Capillary tube geometry in Fig. 18 was tested with various Laplace number for validating the 3D performance of contact angle and solid interface. At the same grid resolution, the 3D computation has same accuracy as 2D, where the interface location is tracked with an accuracy of one cell distance. The change of wall attachment point at $30^{\circ}$ contact angle is tracked in time from initial flat interface shape to the final steady state in Fig. 18. With a modest Laplace number, for example, $L a=100$, the interface shape evolves smoothly without overshooting, but a large Laplace number can cause oscillations in interface shapes before it reaches the steady state solution because the relatively large surface tension and small viscosity create a larger contact line force, and thus movement. Figure 19 shows the 3D sample snapshot of deformed interface shape for given contact angles 30, 90, and $150^{\circ}$. The Cartesian grid is locally adapted dynamically tracking the interface location for effective computation. The streamlines and pressure contour in a plane of 3D domain are shown in Fig. 20.

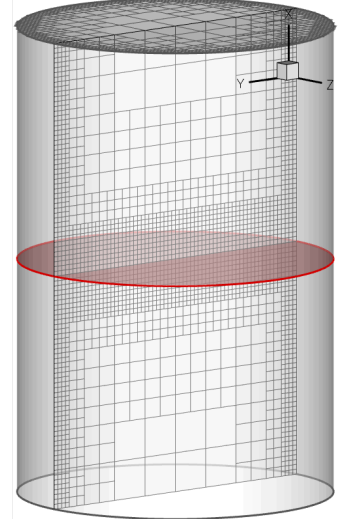

(a) $\mathrm{t}^{*}=0.0$

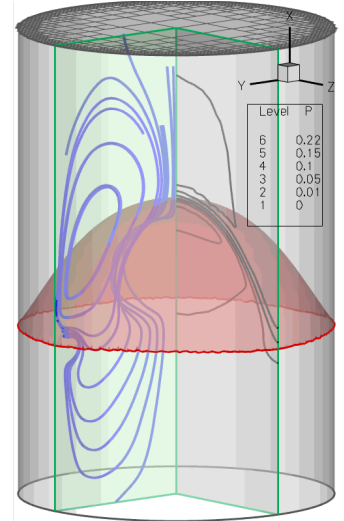

(b) $\mathbf{t}^{*}=\mathbf{2 0 . 0}$

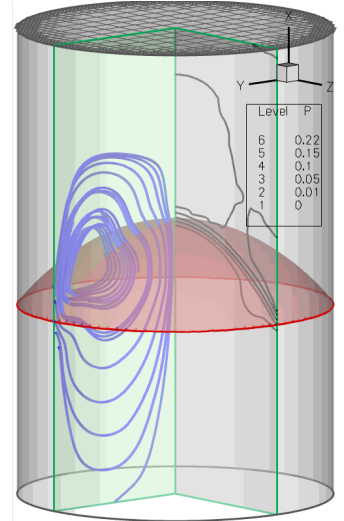

(b) $\mathbf{t}^{*}=\mathbf{4 0 . 0}$

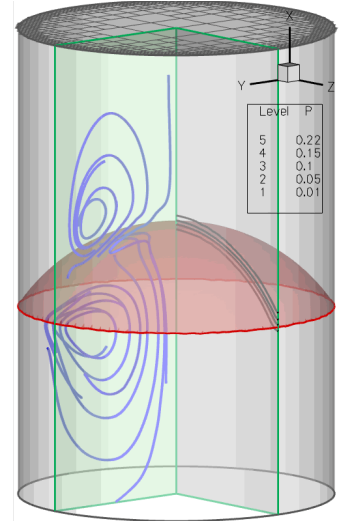

(c) $\mathrm{t}^{*}=\mathbf{1 0 0 . 0}$

Figure 20. Evolution of streamlines and pressure of 3D Capillary tube with $150^{\circ}$ contact angle.

\section{B. The Liquid Plug Flows}

The lung airways are coated with a thin liquid film, which can become thicker during various situations, such as congestive heart failure, asthma, cystic fibrosis, delivery of drugs ${ }^{50}$. This liquid film can become unstable and form airway closure, or liquid plug, which can initiate undesired outcomes for the patient. In addition to having a role of blocking gas exchange, liquid plugs can cause damage to pulmonary epithelial cells because of the additional mechanical stresses they bring ${ }^{51}$. These factors become more significant especially when liquid plug experiences a rupture (reopening of the airway), causing a sudden change in air pressure. Many factors can lead to a rupture, including the liquid properties, gravity, propagation speed, presence of downstream plugs, airway geometry, plug size, and interfacial activity ${ }^{52}$.

In this section, we investigate the dynamics of a liquid plug rupture for a small plug length, where the van der Waals attraction forces significantly affects the dynamics and stability of the plug flow. In particular, the van der Waals forces can be the major source determining the threshold of the rupture. The critical length is determined based on non-dimensional form of Navier-Stokes equations, which is consistent with prior considerations ${ }^{53,54}$ focusing on thin liquid films.

In order to investigate the rupture dynamics and its effects, it is desirable to start the numerical simulation with a steady state solution of the liquid plug. Hence the numerical simulation is handled in two stages of computations; (i) obtaining steady state solutions of liquid plug propagating in an infinitely long channel when no attraction forces are present, and (ii) using the steady state solution with van der Waals potential. The steady state solutions are obtained at various plug lengths to compare our approach with prior studies of Fujioka and Grotberg ${ }^{51}$, who employed a Lagrangian type interface tracking in a $2 \mathrm{D}$ channel. In addition to planar results, we extend their study for axisymmetric computations.

Figure 21 shows the computational setup based on the channel geometry and the liquid plug. Two air-bubbles containing different pressure levels drive the liquid plug at a constant speed. The liquid plug length, denoted by $L_{p}$, is the distance between air bubbles. The liquid film thickness for air bubbles are denoted as $h_{1}$ and $h_{2}$. 


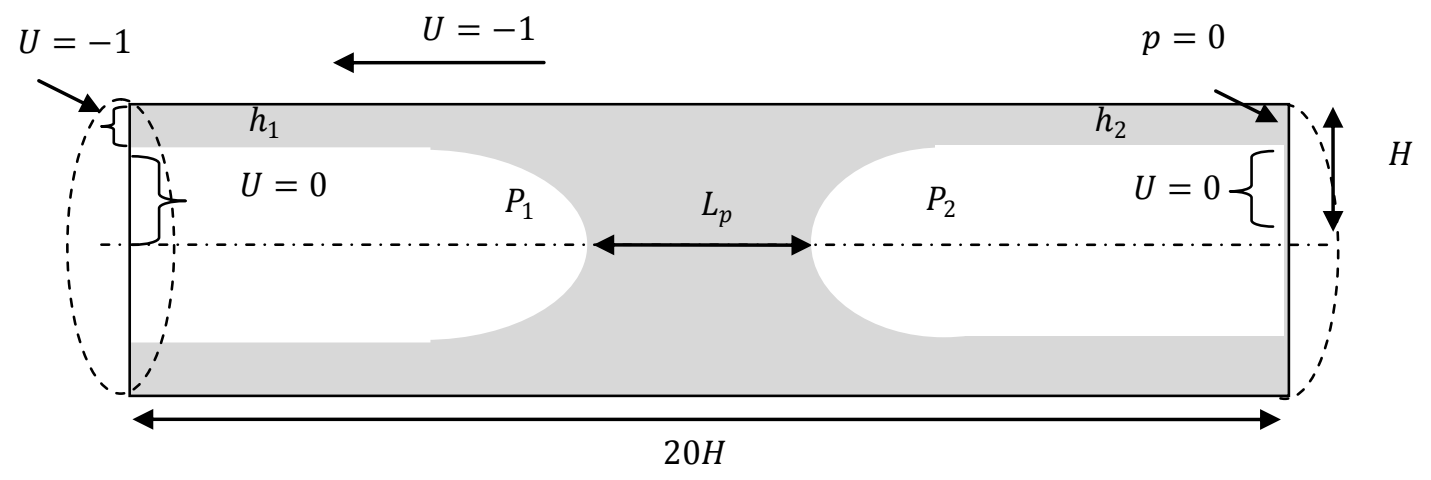

Figure 21. Computational setup and the boundary conditions.

The flow conditions are set by taking the characteristic length as the radius, denoted with $H$, and characteristic velocity as the bubble tip velocity, denoted with $U$. Focusing on the liquid plug region, no-slip wall condition at the pipe wall is employed by changing the computational framework to follow the bubble tip, which propagates at a constant speed. Using the described computational framework, axial velocity at the channel walls is set to negative unity yielding a zero-velocity for the bubble tip. The boundary conditions at the channel entrance and exit are designed to honor the infinitely long channel assumption. No air is allowed to enter or escape from the channel by setting the velocity profiles inside the air bubble to zero. On the other hand, a linear velocity profile is assigned to liquid at the entrance based on velocity profiles on the interface and the no-slip wall. Outflow conditions based on constant pressure leveled at zero is assigned at the channel exit. Lastly, symmetry conditions are considered at the centerline to facilitate half-domain solutions to the planar and axisymmetric numerical simulations.

Based on the parameters presented in Fig. 21, the non-dimensional parameters based on liquid's density and viscosity are given in $\mathrm{Eq}(50)$.

$$
R e=\frac{\rho_{l} H U}{\mu_{l}} ; C a=\frac{\mu_{l} U}{\sigma}
$$

\section{Steady State Liquid Plug Propagation}

The flow properties of steady state plug propagation are obtained via time marching from an initial condition of a zero velocity field and an arbitrary interfacial geometry, which is chosen to be a combination of half circles in the vicinity of the liquid plug and straight lines to resemble infinitely long air bubble with no variation in film thickness.

The first set of steady state solution focus on the two-dimensional channel flow, similar to the numerical study carried by Fujioka and Grotberg ${ }^{51}$. We consider the shape of the plug and the mechanical stresses at the channel wall at $R e=50$ and $C a=0.05$. Three different plug lengths, i.e. $L_{P}=0.5, L_{P}=1.0$ and $L_{P}=2.0$ are studied for validating our approach.

Figure 22 shows the pressure contours and the streamlines for three different values of $L_{P}$ in a two-dimensional channel and axisymmetric circular tube along with the steady state grid distributions. The pressure drop between the air bubbles drives the liquid plug through the channel. A circulation zone at the plug core is observed for each of the solution, causing the film thickness at the upstream of the liquid plug to be at a minimum. This location also marked as the minimum liquid pressure location, which is accompanied by large pressure gradients on each side. A qualitative comparison between the axisymmetric and the planar geometries suggest that the flow structures are similar to determine the critical locations of wall stresses, while the interface shapes, hence the trailing film thickness, $h_{1}$, and the magnitude of the pressure drop across the plug are quantitatively different, as presented in Table 2. For instance, the axisymmetric geometry is observed to have smaller film thickness than the planar cases for all plug lengths. In addition, the trailing film thickness remains the same for plug lengths of 1.0 and 2.0, in spite of an increasing trend seen in planar cases. 

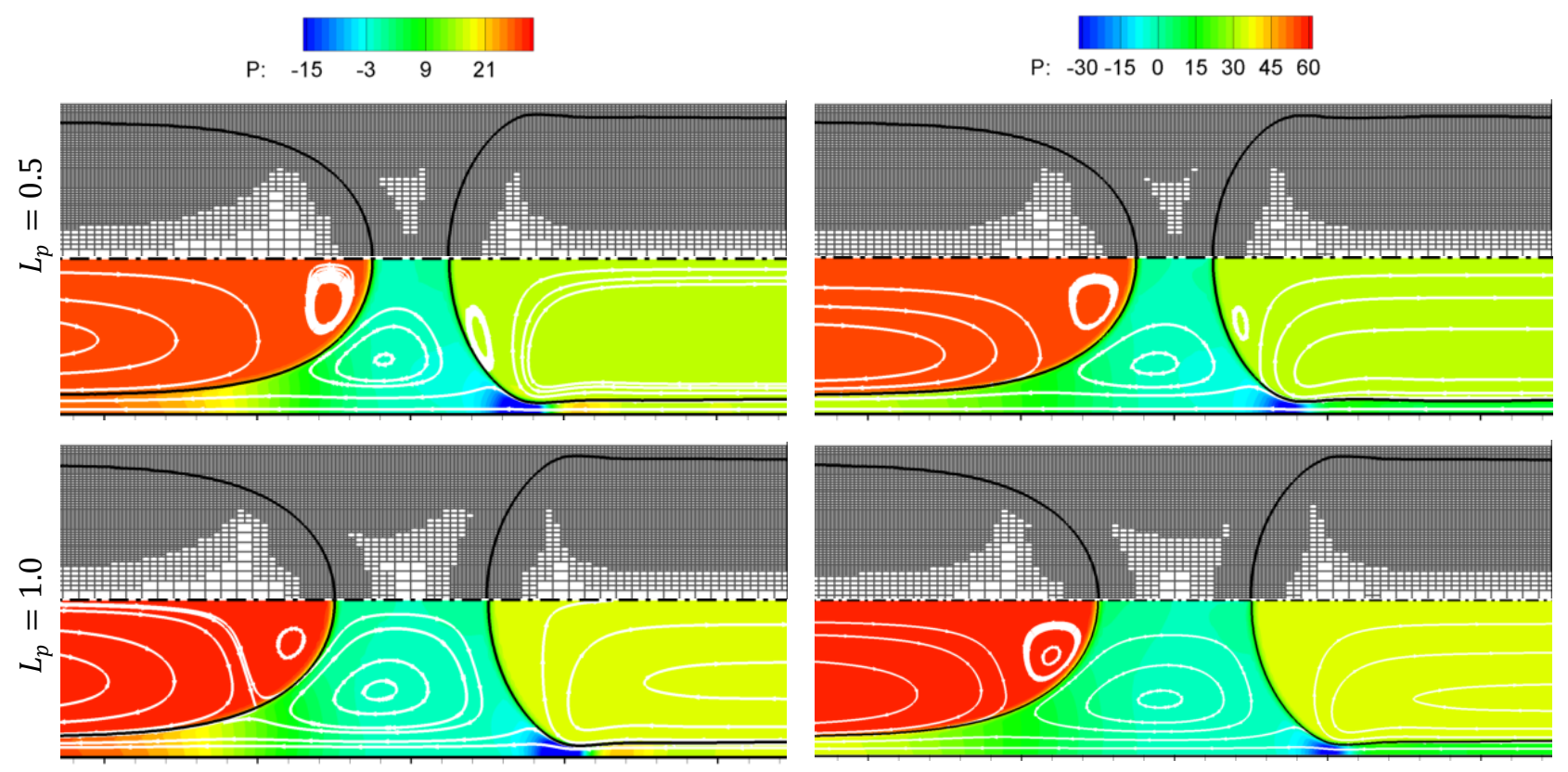

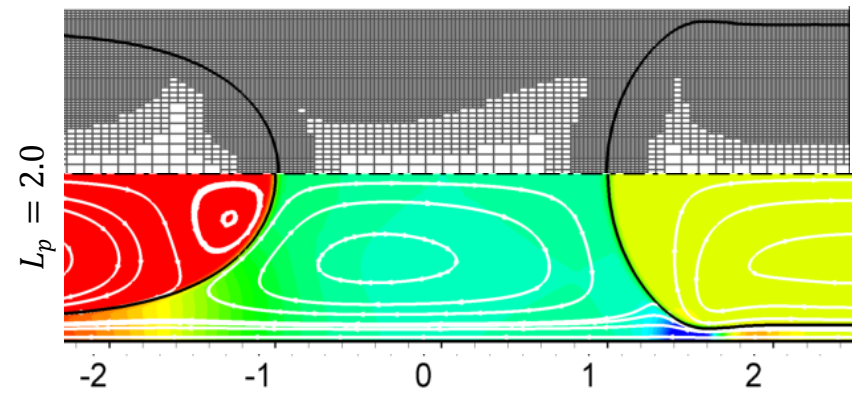

(a)

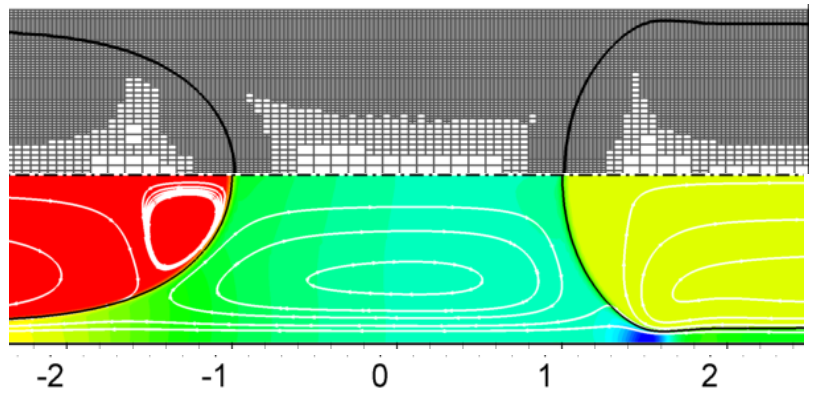

(b)

Figure 22. Two-dimensional steady state solutions of the liquid plug problem with plug lengths: $L p=0.5, L p=1.0$, and $\mathrm{Lp}=\mathbf{2 . 0}$, (a) planar geometry, (b) axisymmetric geometry. Colors indicate pressure levels.

Table 2. Steady state trailing film thickness and the pressure drop across the plug, at $\mathrm{Re}=50$ and $\mathrm{Ca}=0.05$.

\begin{tabular}{|c|c|c|c|ccc|ccc|}
\hline & \multicolumn{3}{|c|}{ Axisymmetric } & \multicolumn{4}{c|}{ Planar } \\
\hline & \multicolumn{2}{|c|}{ Present Computations } & \multicolumn{3}{c|}{ Present Computations } & \multicolumn{3}{c|}{ Fujioka and Grotberg $^{51}$} \\
\hline$L_{p}$ & 0.5 & 1.0 & 2.0 & 0.5 & 1.0 & 2.0 & 0.5 & 1.0 & 2.0 \\
\hline$h_{1}$ & 0.111 & 0.115 & 0.115 & 0.114 & 0.119 & 0.123 & 0.113 & 0.119 & 0.123 \\
\hline$\Delta P / L_{p}$ & 46.0 & 24.0 & 14.7 & 21.7 & 12.0 & 7.1 & 23.0 & 13.0 & 8.0 \\
\hline
\end{tabular}

In Table 2, the film thickness length and the pressure drop across the plug for planar geometries are compared with the prior numerical study by Fujioka and Grotberg ${ }^{51}$. Their numerical approach adopts a Lagrangian method to identify the moving air bubble shape with a single fluid formulation, which assumes constant pressure inside the air bubble. In present approach, this assumption is replaced by assigning material properties to air, i.e. density ratio as 50 and viscosity ratio as 10 . Despite the difference in formulations, both approaches agree on the characteristics of the flow. For instance, both 2D planar results show that the steady state trailing film thickness, $h_{1}$, decreases with a decreasing plug length for the two-dimensional channel and the steady state pressure drop across the plug, $\Delta P / L_{P}$, shows the same decreasing trend for larger plug lengths. Quantitatively, $h_{1}$ and $\Delta P / L_{P}$ are within $1 \%$ and $10 \%$, respectively. The discrepancy in $\Delta P / L_{P}$ can be related to the difference in single and two-fluid formulation which in return affects the interface shape at the region where the film thickness is at minimum and the wall shear stresses have the steepest gradient. At this location, we obtained the minimum peak value of the non-dimensional wall shear stress, defined as $\tau=C a \partial u / \partial y(y=-1)$, at $R e=40$ as -0.19 whereas Fuijoka and Grotberg ${ }^{51}$ reported it to be -0.41. As illustrated in Fig. 23, both formulations agree with each other at other locations. 
Figure 24 illustrates the wall shear stress distribution for the planar geometry for various plug lengths at $R e=50$ and $C a=0.05$. Because the flow is uniform for the liquid film at locations away from the plug, the shear stress becomes zero. For all plug lengths, the shear stress starts to increase at the trailing liquid film region, reaching a local maximum. Then the shear stress starts to decay as a result of the right bubble interface shape. The shear stress at the core of the liquid plug is almost constant for the plug length, $L_{p}=2.0$, which asymptotically approaches to a value of $3 \mathrm{Ca}\left(1-h_{1}\right)$ considering the velocity profile of $2 \mathrm{D}$ Poiseuille flow as $U=-1+3 / 2\left(1-h_{1}\right)\left(1-y^{2}\right)$. This result is consistent with our prior study ${ }^{14}$, in which plug length is considered to be very large, i.e. $L_{P} \rightarrow \infty$. For plug lengths, $L_{p}=0.5$ and $L_{p}=1.0$, this region is no longer flat as a result of the existence of upstream air bubble. The maximum shear stress is observed at the location where the film thickness is at minimum. This is followed

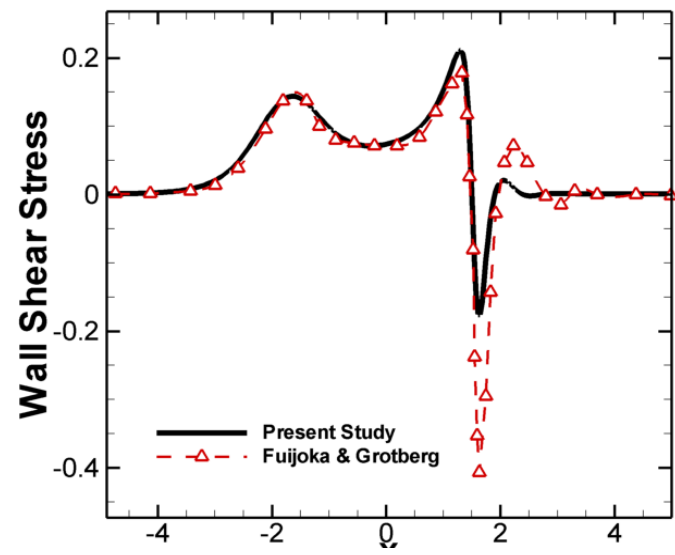

Figure 23. Steady state wall shear stress obtained by the present study and Fuijoka \& Grotberg ${ }^{50}$. $R e=40, C a=0.05$ and $L_{p}=2.0$. by a sharp change in shear stress resulting in a oscillations in the upstream liquid film region until zero stress is attained further away from the plug core. The pressure distribution along the wall is also shown in Fig. 25. Similar to the shear stress values, the peak magnitudes of the wall shear stresses do not change with the plug length, while the wall shear stress at the plug core is larger for smaller plug lengths.

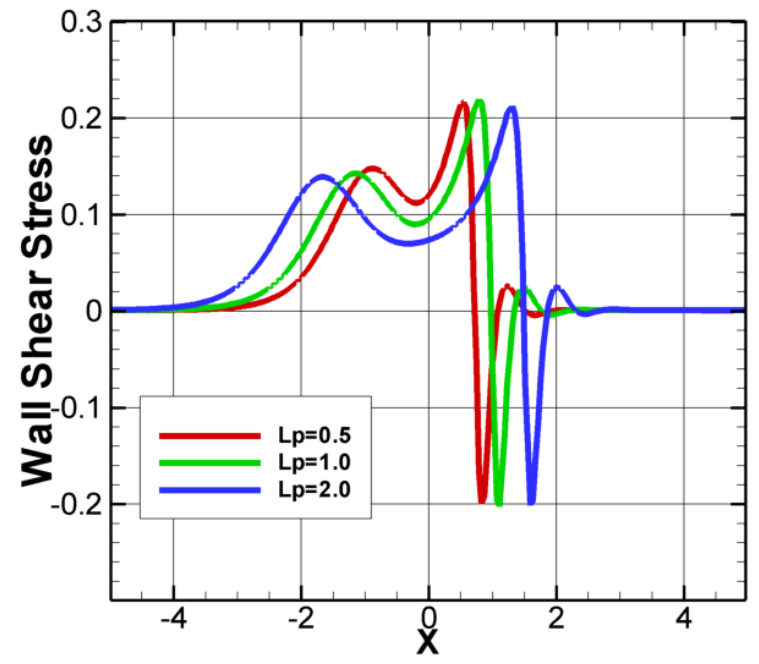

Figure 24. Steady state wall shear stress distibution for vaious plug length at $\mathbf{R e}=\mathbf{5 0}$.

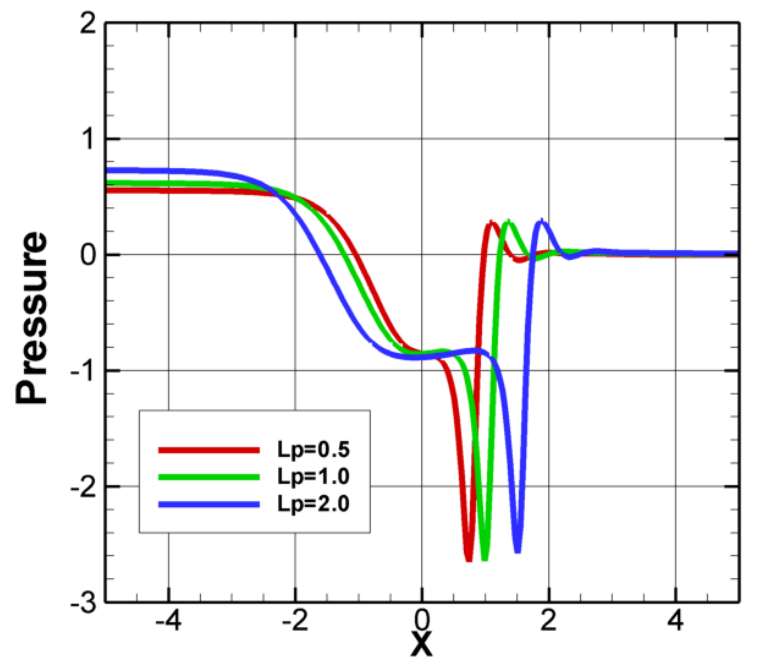

Figure 25. Steady state dimensional pressure scaled with the surface tension $\left(P^{*}=\mathrm{CaP}\right)$ disribution for various plug lengths at $\mathbf{R e}=\mathbf{5 0}$.

\section{The van der Waals Effects}

The main focus of the present study is to capture the flow dynamics and the stresses occurring at the channel walls during the airway reopening by numerically simulating the plug rupture. To investigate the rupture dynamics, we initiate flow using the steady state solutions obtained in the absence of the van der Waals attraction forces. These forces are the main mechanism of a reopening as they are reversely proportional with distance between the air bubbles. The film thickness defined earlier corresponds to the liquid plug length. Hence, the smaller the plug length, the more dominant they become over other forms of forces, i.e. surface tension. Eventually the liquid plug will rupture causing bubble interfaces to merge removing the airway blockage.

We consider the van der Waals attraction forces on the axisymmetric configuration for the plug length of $L_{P}=0.5$. The potential of the attraction forces at an interface location is defined based on the minimum distance to another fluid interface. This distance is not only used to compute the potential but also utilized to determine the time of the rupture, at which the film becomes unstable. Once the liquid plug length becomes smaller than a critical plug length, rupture is numerically initiated. The critical plug length is determined by the balance between the surface tension and the attraction forces using Eq. (33). The attraction forces defined by the Hamaker constant, $A$, is introduced to the computations as unity, whereas all other parameters including boundary conditions are kept the 
same as the steady state cases. According to these parameters, non-dimensional time and critical liquid plug length that would cause a rupture read:

$$
t=\frac{A \rho}{\sigma \mu} t^{*} ; \quad h_{\text {critical }}=\sqrt{\frac{A}{\sigma}}=0.22
$$
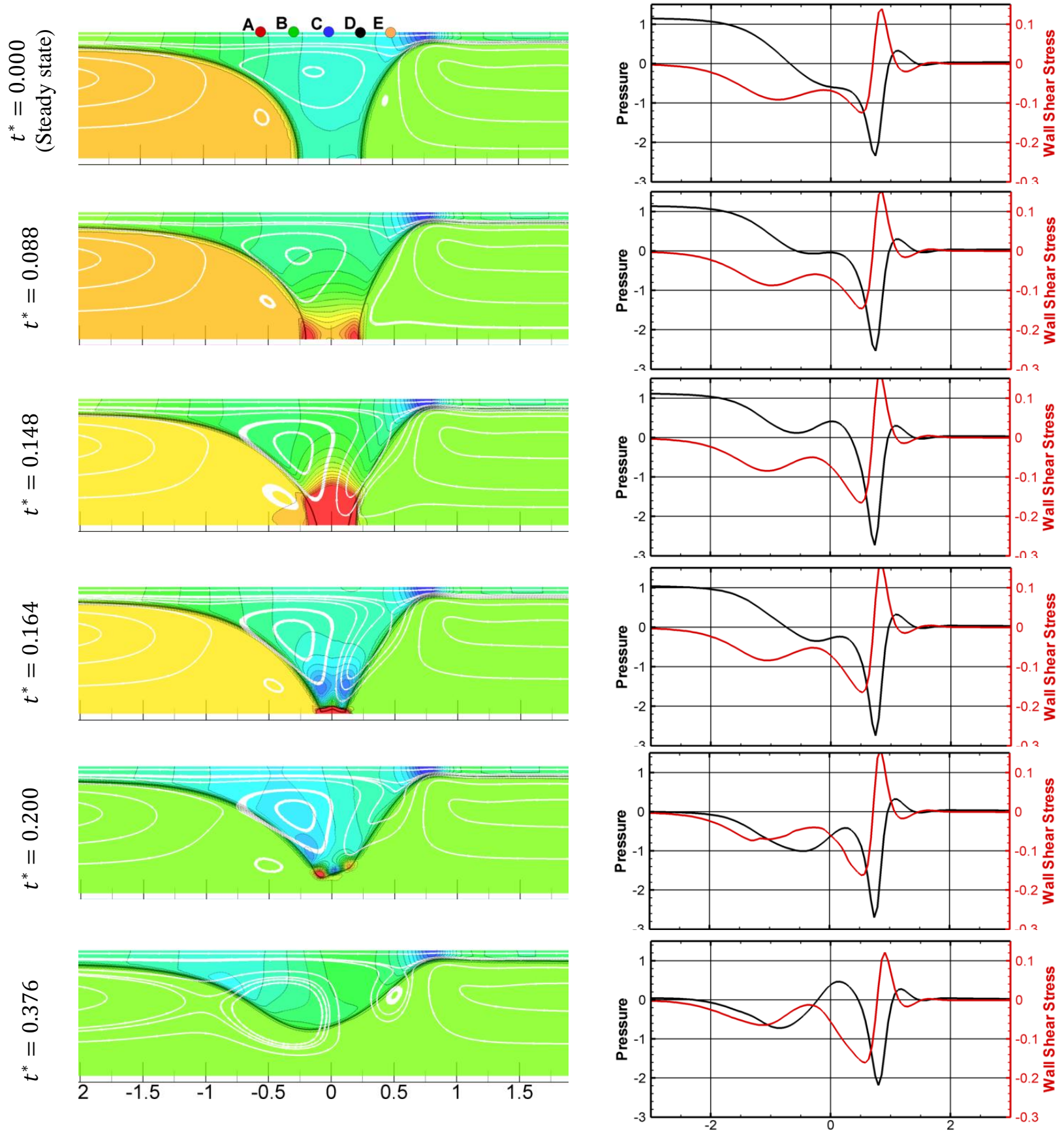

(a)

(b)

Figure 26. Time instants of the flow field during the reopening of the airway at $\mathrm{Re}=50, \mathrm{Ca}=0.05$ with an initial plug length of 0.5. (a) Pressure contours levels (color) and streamlines (white lines). (b) Wall pressure and wall shear stress. 
Figure 26 shows the time history of the pressure contours and streamlines before and after the rupture. The rupture is observed to happen between $t^{*}=0.148$ and $t^{*}=0.164$. Before rupture happens, the pressure starts to build up at the liquid plug as its length kept decreasing. At the rupture time, $t^{*} \cong 0.16$, the length goes below the critical plug length and the interfaces experience a topological change, which causes a sudden change in pressure. Afterwards, the surface tension becomes locally dominant in this region, causing the freshly merged interface to move towards the channel walls. The sudden change in pressure level due to reopening is felt at the walls. This is illustrated using the time variation plot of wall shear stress and wall pressure at five different locations, marked in Fig. 26 as $A$ to $E$, are shown in Figs. 27 and 28.

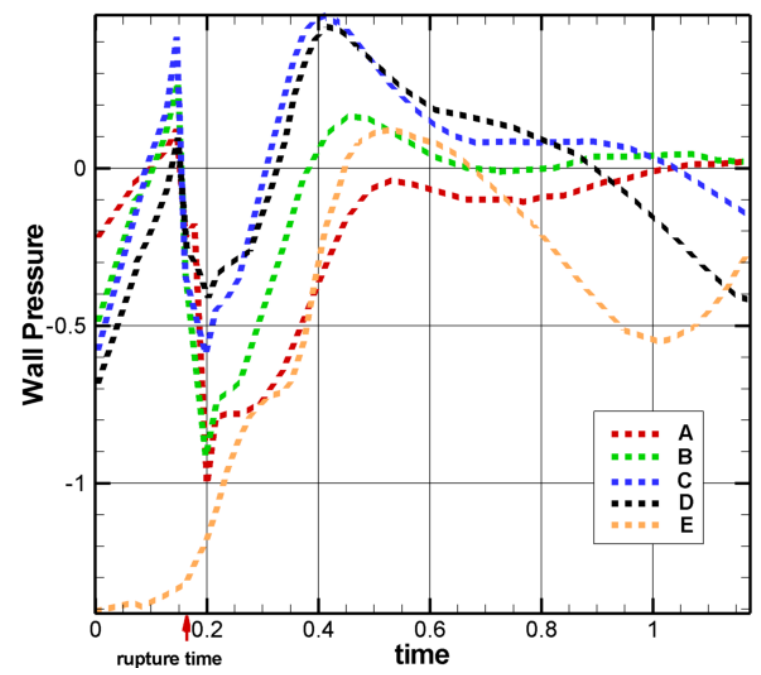

Figure 27. Time history of wall pressure during the rupture of a liquid plug with a length of 0.5 .

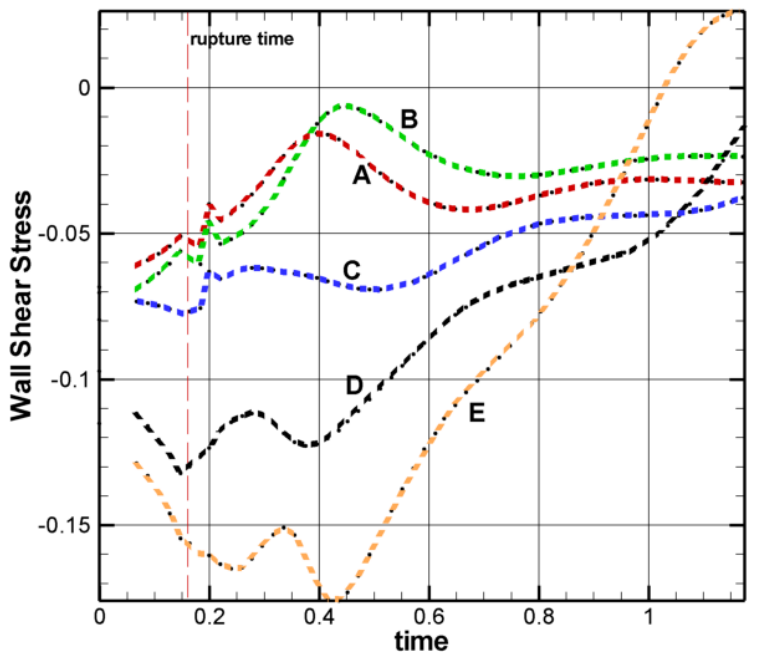

Figure 28. Time history of wall shear stress during the rupture of a liquid plug with a length of 0.5 .

In Fig. 27, the abrupt change in pressure is directly observed by all probe locations except point $E$, which is located near the minimum film thickness. Before the rupture, the wall pressure in the vicinity of the liquid plug $(B$ $D$ ) is observed to increase more rapidly than the location at liquid film ( $A$ and $E$ ). This is due to the inclusion of the van der Waals attraction forces applied at the liquid plug region. As a result, the gradient of pressure changes sign between $B$ and $C$. On the other hand, the wall shear stress tends to decrease for $A$ and $B$ while its magnitude grows at points $C, D$, and $E$. At the time of the rupture, the sudden change in pressure at point $B$ is the largest, suggesting large normal stresses acting at the channel wall. On the other hand, shear stress at $B$ experiences a small increase followed by a gradual decay until $t=0.41$, when the shear stress at location $E$ reaches to its maximum at $t=0.41$ after which rapidly decays with an increasing film thickness at this region. For a period of time, the gradient of pressure is reversed for $A-B(0.2<t<1.17), B-C(0.17<t<0.98)$, and $C-D(0.42<t<0.81)$. Overall, during a liquid plug rupture the critical locations at the airway walls is observed to be in the vicinity of point $\mathrm{B}$, where a sudden change in normal stresses occur, and point $\mathrm{E}$ where the shear stress reaches at its maximum as a result of the interface shape change causing change in the film thickness.

\section{Spacecraft Fuel Tank Dynamics}

The dynamics of the liquid fuel contained in a tank is of interest especially for spacecraft applications. Considering micro-gravity conditions, capillary and viscous effects can become as significant as inertia effects, as opposed to normal gravity conditions. In such conditions, the flow characteristics may change rapidly in response to the spacecraft motion as a result of altered magnitude and direction of the body forces. These factors not only determine the amount of fuel delivered to the combustion chamber but also influence the spacecraft dynamics because the flow motion in the tank can disturb the dynamics of the vehicle by changing its center of mass ${ }^{55}$.

\section{Draining Tank Flow}

The liquid fuel draining process depends on many factors, i.e. tank geometry, acceleration, surface tension, pressure difference applied and the material properties of the liquid fuel ${ }^{14,15}$. The effects of these parameters have 
been studied experimentally ${ }^{56,57}$ under the effects of axial acceleration. When the acceleration or the gravitational force is aligned with axial axis of a symmetric fuel tank, the problem can be tacked in an axisymmetric formulation of the Navier-Stokes equations ${ }^{14,15}$. On the other hand, when the direction of the acceleration is different, the dynamics can only be captured using full three-dimensional approach.

In the present study, we first examine the validity of our developed strategy in 3D configuration using our previously reported results ${ }^{14,15}$. Then, we test fuel tank draining case at $45^{\circ}$ tilted micro-gravity. Weber number, Bond number and Reynolds number are used to study the draining flow in a micro-gravity condition.

$$
\begin{gathered}
\text { Weber Number, We }=\frac{\rho Q^{2}}{\pi^{2} \sigma R^{3}} \\
\text { Bond Number, Bo }=\frac{W e}{F r}=\frac{\rho g R^{2}}{\sigma} \\
\text { Reynolds Number, } R e=\frac{\rho U(2 R)}{\mu}
\end{gathered}
$$

In these equations, $\rho$ is the density of the liquid, $Q$ is the volume flow rate, $\sigma$ is the surface tension of the interface between gas and liquid, $g$ is the gravitational acceleration, and $R$ is the characteristic length of the fuel tank, which is taken as the radius for cylindrical geometry in this study. Accordingly, non-dimensional time is defined as $t^{*}=t Q / \pi R^{3}$.

Figure 29 shows the computational geometry configuration used for the verification of the three-dimensional computations. An axisymmetric fuel tank with a hemispherical bottom is considered to compare 3D computation with axisymmetric one. The initial fluid interface shape is assumed as circular arc with the given contact angle, $45^{\circ}$, at the tank wall. Bond number, Weber number, and Reynolds number are considered as 5, 0.97, and 216, respectively. Figure 30 illustrates that non-dimensional centerline and wall attachment location of the interface for 3D computations are in agreement with the axisymmetric computations. The snapshots of adaptive grids and pressure contour for 3D computation are shown in Fig. 31, where maximum of 21 grid points are used along radius of tank. In spite of the fact that there are only 4 cells across the outlet pipe, the core dynamics of the draining process is captured.

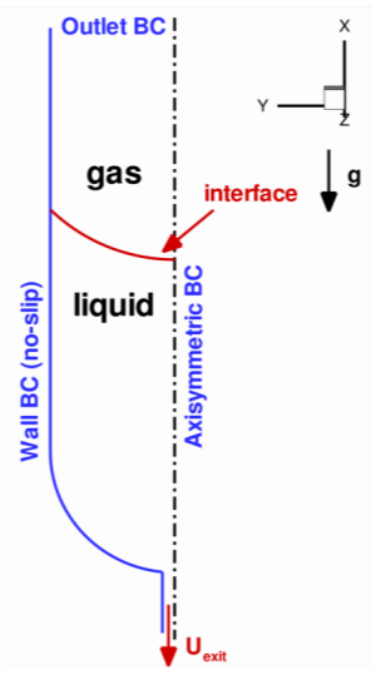

(a)

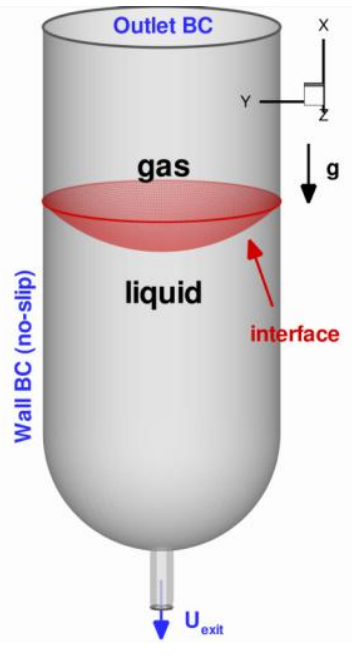

(b)

Figure 29. The computational geometry configuration of draining fuel tank with hemispherical bottom. (a) axisymmetric domain, (b) 3D computational domain

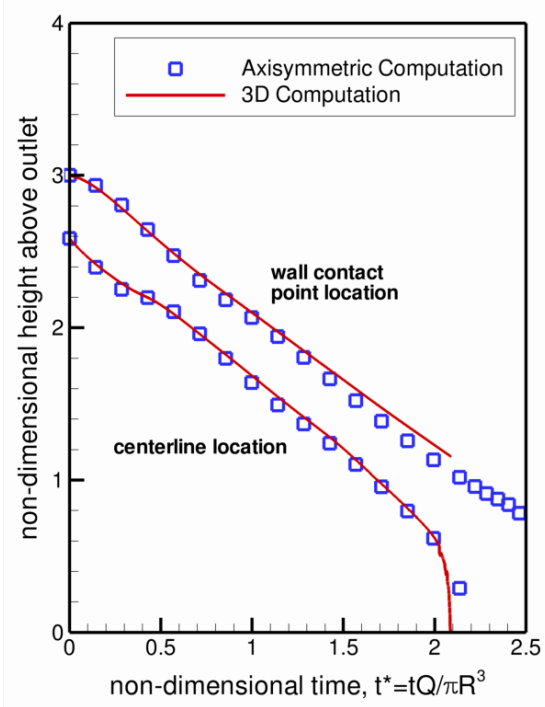

Figure 30. The comparison between axisymmetric and three dimensional computation for draining tank flow simulation. $B o=5, W e=0.97$, and $R e=216$. 


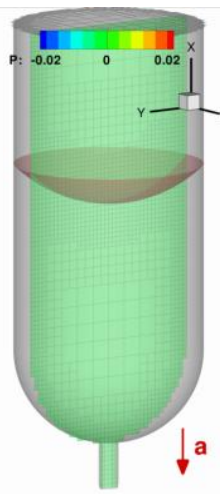

(a) $\mathbf{t}^{*}=\mathbf{0 . 0}$

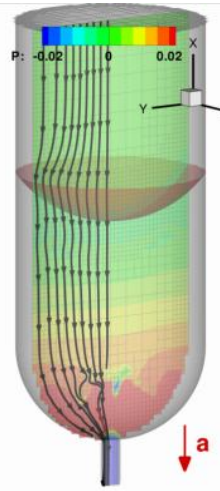

(b) $t *=0.2$

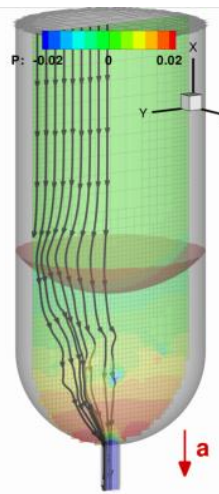

(c) $\mathrm{t}^{*}=\mathbf{1 . 0}$

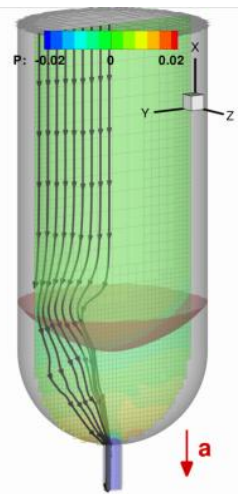

(d) $\mathbf{t}^{*}=1.6$

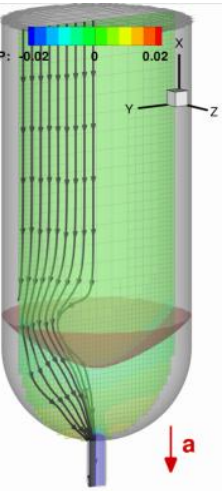

(e) $\mathrm{t}^{*}=1.8$

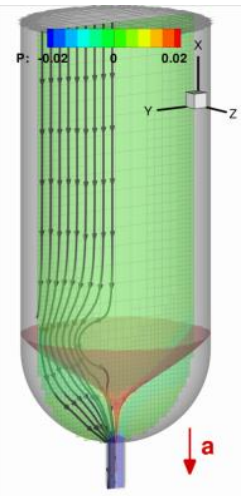

(f) $\mathrm{t}^{*}=\mathbf{2 . 0}$

Figure 31. The snapshots of 3D liquid fuel draining flow under axial-direction micro-gravity. Grids are locally adapted around interface in time. Black lines represent streamlines and colored contour shows pressure distribution.

To illustrate the 3D capabilities, the draining fuel tank simulation is conducted at a $45^{\circ}$ tilted micro-gravity condition in Fig. 32. The ratio between the outlet radius, $r$, and the tank radius, $R$, is $1 / 10$, and the tank height is 4.0 times of tank radius. All simulations are conducted in a micro-gravitational environment with $1.5 \%$ of normal gravity. The initial fluid interface shape is assumed as a circular arc with the given contact angle, $45^{\circ}$, at the tank wall. Bond number, Weber number, and Reynolds number are considered as 5, 0.97, and 21.6, respectively. The computational geometry configuration is shown in Fig. 32. The complex 3D tank wall is represented by solid interface on a Cartesian computational grid. Figure 33 shows the snapshots of adaptive grid and pressure contour plot. Similar to the axial gravity case, maximum of 21 grid points along the tank radius is employed, which corresponds to 4 grid cells per the diameter of the outlet pipe.

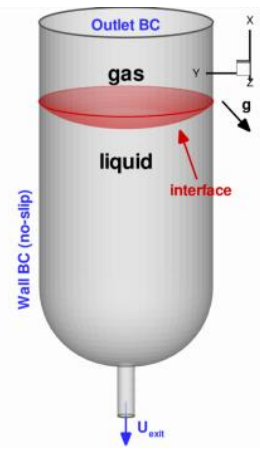

Figure 32. The computational geometry configuration of 3D draining fuel tank with tilted acceleration. $(\mathrm{Bo}=5, \mathrm{We}=0.97$, and $\mathrm{Re}=21.6)$

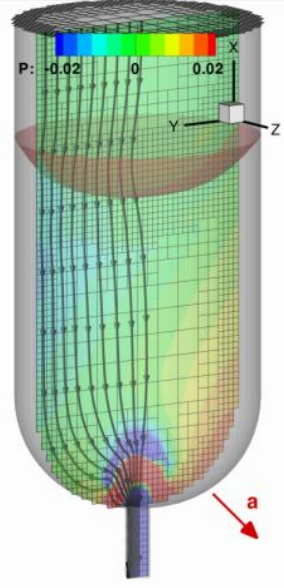

(a) $\mathrm{t}^{*}=\mathbf{0 . 1 5}$

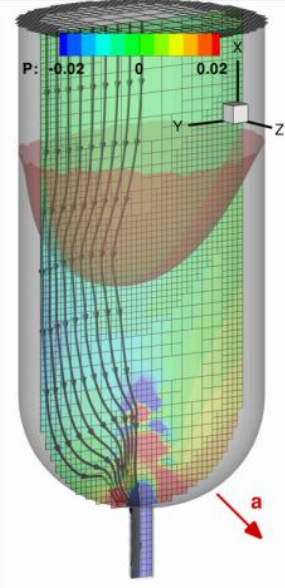

(b) $\mathrm{t}^{*}=0.60$

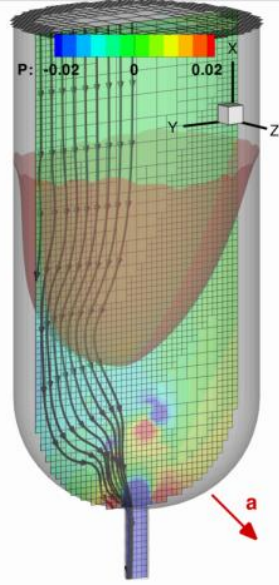

(c) $\mathrm{t}^{*}=\mathbf{1 . 0 5}$

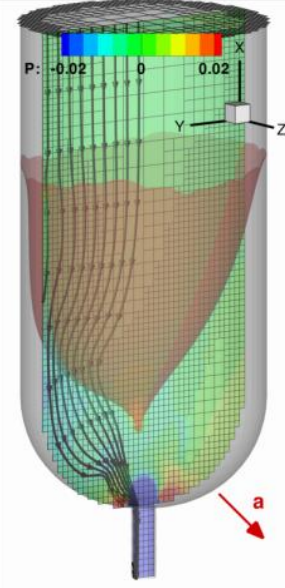

(d) $\mathbf{t}^{*}=1.28$

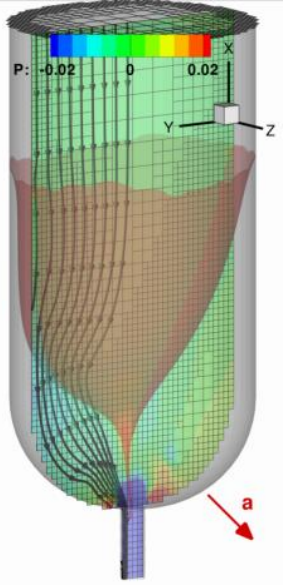

(e) $\mathbf{t}^{*}=1.33$

Figure 33. The snapshots of $3 \mathrm{D}$ fuel tank draining with $45^{\circ}$ tilted gravitational acceleration. The magnitude of acceleration is $0.015 \mathrm{~g}_{0}$, and $45^{\circ}$ contact angle is applied on the wall. $(B o=5, W e=0.97$, and $R e=21.6)$ 


\section{Sloshing liquid motion in a spacecraft fuel tank}

The sloshing waves on the liquid fuel surface can influence the fuel draining dynamics affecting vapor ingestion and liquid fuel residuals. In addition, it can change the whole spacecraft dynamics due to the movement of center of mass of the liquid fuel during landing and/or docking maneuver. At normal or higher gravitational acceleration during powered flight, the fluid motion is remained damped, and shows moderate flow motion. On the other hand, even smaller potential energy change can cause large movements under microgravity conditions. For example, the sudden engine cutoff transforms the potential energy of liquid at a higher acceleration into kinetic energy, and thus, large sloshing fluid motion. Understanding the flow motions in a liquid fuel tank, and its influence on the spacecraft dynamics are crucial for spacecraft applications.

The present study considers the pure sloshing motion of a liquid fuel in a spacecraft tank. Figure 34(a)

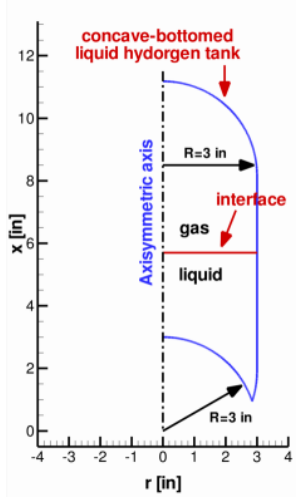

(a)

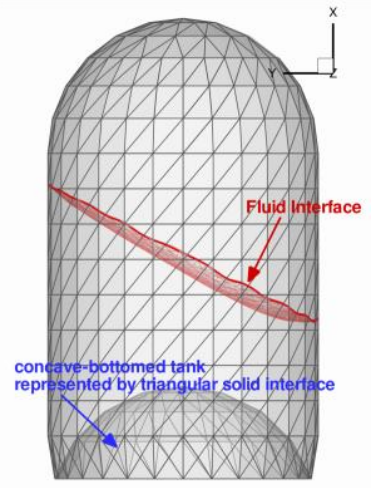

(b)

Figure 34. The computational geometry configuration of draining fuel tank with hemispherical bottom. (a) axisymmetric domain, (b) 3D computational domain shows the configuration of the scale model of the Saturn V/S-IVB liquid hydrogen tank experimented by Toole et al. ${ }^{58}$ This complex concave-shaped tank is represented by the triangular solid interface elements in Fig. 34(b) for 3D computation on the stationary Cartesian grids. Figure 35 shows the flow field and instantaneous interface shapes during a sloshing flow inside this particular geometry undergoing a sudden change of direction of acceleration at $1 \%$ of the earth gravity level. The applied acceleration is initially $45^{\circ}$ tilted for the axis in Fig. 35(a), where, the deformed interface with $60^{\circ}$ contact angle between liquid and gas are shown. At $t^{*}=0.0$, the applied acceleration is abruptly changed into axial direction, and the difference of potential energy makes sloshing motion. Bond number, which is defined as the ratio of gravitational force to surface tension forces, $B o=24$ is used in this simulation.

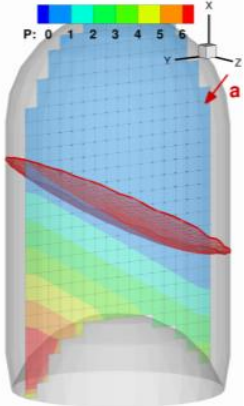

(a) $\mathrm{t}^{*}=0.0$

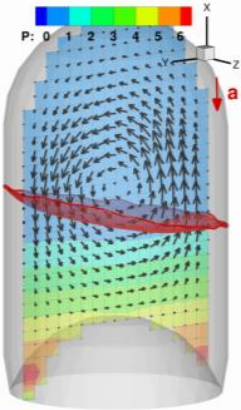

(b) $\mathbf{t}^{*}=1.0$

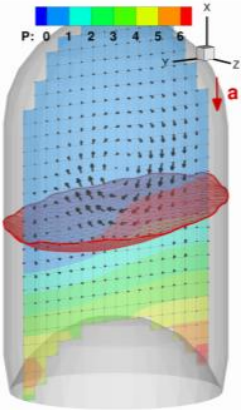

(c) $\mathrm{t}^{*}=2.2$

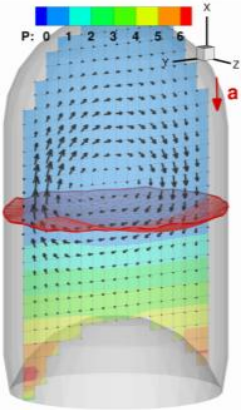

(d) $\mathrm{t}^{*}=3.4$

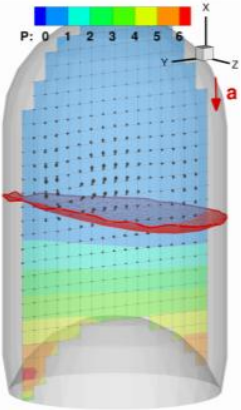

(e) $\mathrm{t}^{*}=4.2$

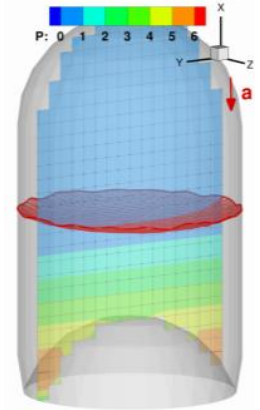

(f) $\mathbf{t}^{*}=\mathbf{2 0 . 0}$

Figure 35. Sloshing motion of liquid fuel in a concave-bottomed tank with a sudden change of accelerational direction. Velocity vector and colored pressure contour are represented.

\section{Summary and Conclusions}

In this paper, we report our recent efforts in developing a multi-scale three-dimensional adaptive grid method for multiphase flow problems. The method utilizes marker points representing a triangulated surface for both tracking interfaces separating fluid constituents and arbitrarily shaped solid geometries. The transport equations are solved on the Cartesian grid with automated local grid adaptation to capture the flow features. The triangular mesh representing the Lagrangian framework is free to move on the Cartesian grid that represents the Eulerian framework. These components are coupled together to capture interfacial flow dynamics for various applications. In summary, the following key ingredients are developed and incorporated: 
(i) The marker-based data structure that allows contact lines on computational boundaries and other marker based interfaces

(ii) Surface tension forces on interfaces separating fluid phases, as well as on contact lines where fluid-liquidgas phases all meet,

(iii) Modeling marker based surface as a solid geometry, to allow Cartesian grid based computations around two- and three- dimensional irregular object.

(iv) Indicator function based on distance formulation to distinguish materials, and aid cost of computations relevant to geometric calculations.

(v) Multiple physical mechanisms including surface tension, van der Waals effects in the interface region, along with the full Navier-Stokes equations.

To highlight these features, the following case studies are presented:

(i) Capillary flows with various Laplace numbers and contact conditions.

(ii) Spacecraft fuel tank flow problems with gravitational effects and varied control parameters including the capillary and Bond numbers.

(iii) The liquid plug problem, motivated by issues associated by drug delivery into the lungs, is investigated using the present two-fluid formulation. The combined surface tension and van der Waals effects, interacting with different plug lengths and flow parameters, leading to rich physical phenomena including topological changes and distinct flow characteristics.

\section{Acknowledgments}

The work reported in this paper has been partially supported by NASA Constellation University Institutes Program (CUIP), Claudia Meyer and Jeff Rybak program managers. We have benefitted from communication with Jim Grotberg and Hideki Fujioka of the University of Michigan while investigating the liquid plug flow problems.

\section{References}

${ }^{1}$ Uzgoren, E., R. Singh, J. Sim, and W. Shyy, Computational modeling for multiphase flows with spacecraft application. Progress in Aerospace Sciences, 2007. 43: p. 138-192.

${ }^{2}$ Shyy, W., H.S. Udaykumar, M.M. Rao, and R.W. Smith, Computational Fluid Dynamics with Moving Boundaries. Taylor \& Francis, Washington, DC, (1996, revised printing 1997, 1998\&2001). 2007, New York: Dover.

${ }^{3}$ Osher, S. and R.P. Fedkiw, Level Set Methods and Dynamic Implicit Surfaces. 2002: Springer.

${ }^{4}$ Scardovelli, R. and S. Zaleski, Direct numerical simulation of free-surface and interfacial flow. Annual Review of Fluid Mechanics, 1999. 31(1): p. 567-603.

${ }^{5}$ Tryggvason, G., B. Bunner, A. Esmaeeli, N. Al-Rawahi, W. Tauber, J. Han, Y.J. Jan, D. Juric, and S. Nas, A front-tracking method for the computations of multiphase flow. Journal of Computational Physics, 2001. 169(2): p. 708-759.

${ }^{6}$ Tryggvason, G. and A. Prosperetti, eds. Computational Methods for Multiphase Flow. 2007, Cambridge University Press.

${ }^{7}$ Wasekar, V.M. and R.M. Manglik, Short-time-transient surfactant dynamics and Marangoni convection around boiling nuclei. Journal of Heat Transfer, 2003. 125(5): p. 858-866.

${ }^{8}$ Perot, B. and R. Nallapati, A moving unstructured staggered mesh method for the simulation of incompressible free-surface flows. Journal of Computational Physics, 2003. 184(1): p. 192-214.

${ }^{9}$ Losasso, F., R. Fedkiw, and S. Osher, Spatially adaptive techniques for level set methods and incompressible flow. Computers and Fluids, 2006. 35: p. 995-1010.

${ }^{10}$ Francois, M.M., S.J. Cummins, E.D. Dendy, D.B. Kothe, J.M. Sicilian, and M.W. Williams, A balanced-force algorithm for continuous and sharp interfacial surface tension models within a volume tracking framework. Journal of Computational Physics, 2006. 213(1): p. 141-173.

${ }^{11}$ Enright, D., F. Losasso, and R. Fedkiw, A fast and accurate semi-Lagrangian particle level set method. Computers and Structures, 2005. 83(6-7): p. 479-490.

${ }^{12}$ Sussman, M., K.M. Smith, M.Y. Hussaini, M. Ohta, and R. Zhi-Wei, A sharp interface method for incompressible twophase flows. Journal of Computational Physics, 2007. 221(2): p. 469-505.

${ }^{13}$ Chang, C.H. and M.S. Liou, A robust and accurate approach to computing compressible multiphase flow: Stratified flow model and AUSM+-up scheme. Journal of Computational Physics, 2007. 225(1): p. 840-873.

${ }^{14}$ Uzgoren, E., J. Sim, and W. Shyy. Computations of multiphase fluid flows using marker-based adaptive, multilevel Cartesian grid Method. in 45th AIAA Aerospace Sciences Meeting and Exhibit. 2007. Reno, NV.

${ }^{15}$ Uzgoren, E., R. Singh, J. Sim, and W. Shyy. A Unified Adaptive Cartesian Grid Method for Solid-Multiphase Fluid Dynamics with Moving Boundaries. in 18th AIAA Computational Fluid Dynamics Conference. 2007. Miami, FL. 
${ }^{16}$ Glimm, J., M.J. Graham, J. Grove, X.L. Li, T.M. Smith, D. Tan, F. Tangerman, and Q. Zhang, Front tracking in two and three dimensions. Comput. Math. Appl, 1998. 35(1).

${ }^{17}$ Glimm, J., J.W. Grove, X.L. Li, and D.C. Tan, Robust Computational Algorithms for Dynamic Interface Tracking in Three Dimensions. SIAM Journal on Scientific Computing, 1999. 21(6): p. 2240-2256.

${ }^{18}$ Singh, R. and W. Shyy, Three-dimensional adaptive Cartesian grid method with conservative interface restructuring and reconstruction. Journal of Computational Physics, 2007. 224: p. 150-167.

${ }^{19}$ Shyy, W., Multiphase computations using sharp and continuous interface techniques for micro-gravity applications. Comptes rendus. Mecanique 2004. 332(5-6): p. 375-386.

${ }^{20}$ Peskin, C.S., The immersed boundary method. Acta Numerica, 2003. 11: p. 479-517.

${ }^{21}$ Francois, M. and W. Shyy, Computations of Drop Dynamics with the Immersed Boundary Method, Part 1: Numerical Algoritm and Buoyancy-Induced Effect. Numerical Heat Transfer: Part B: Fundamentals, 2003. 44(2): p. 101-118.

${ }^{22}$ Ye, T., R. Mittal, H.S. Udaykumar, and W. Shyy, An accurate Cartesian grid method for viscous incompressible flows with complex immersed boundaries. Journal of Computational Physics, 1999. 156(2): p. 209-240.

${ }^{23}$ Leveque, R.J. and Z. Li, The Immersed Interface Method for Elliptic Equations with Discontinuous Coefficients and Singular Sources. SIAM Journal on Numerical Analysis, 1994. 31(4): p. 1019-1044.

${ }^{24} \mathrm{Li}$, Z. and M.C. Lai, The Immersed Interface Method for the Navier-Stokes Equations with Singular Forces. Journal of Computational Physics, 2001. 171(2): p. 822-842.

${ }^{25}$ Liu, X.D., R.P. Fedkiw, and M. Kang, A boundary condition capturing method for Poisson's equation on irregular domains. Journal of Computational Physics, 2000. 160(1): p. 151-178.

${ }^{26}$ Kang, M., R.P. Fedkiw, and X.D. Liu, A Boundary Condition Capturing Method for Multiphase Incompressible Flow. Journal of Scientific Computing, 2000. 15(3): p. 323-360.

${ }^{27}$ Marella, S., S. Krishnan, H. Liu, and H.S. Udaykumar, Sharp interface Cartesian grid method I: An easily implemented technique for 3D moving boundary computations. Journal of Computational Physics, 2005. 210(1): p. 1-31.

${ }^{28}$ Aftosmis, M.J., Solution adaptive Cartesian grid methods for aerodynamic flows with complex geometries. Lecture notes, von Karman Institute for Fluid Dynamics, Series, 1997. 2.

${ }^{29}$ Yang, J. and E. Balaras, An embedded-boundary formulation for large-eddy simulation of turbulent flows interacting with moving boundaries. Journal of Computational Physics, 2006. 215(1): p. 12-40.

${ }^{30}$ Gilmanov, A. and F. Sotiropoulos, A hybrid Cartesian/immersed boundary method for simulating flows with $3 D$, geometrically complex, moving bodies. Journal of Computational Physics, 2005. 207(2): p. 457-492.

${ }^{31}$ Shin, S., S.I. Abdel-Khalik, and D. Juric, Direct three-dimensional numerical simulation of nucleate boiling using the level contour reconstruction method. International journal of multiphase flow, 2005. 31(10-11): p. 1231-1242.

${ }^{32}$ Juric, D. and G. Tryggvason, Computations of boiling flows. Int. J. Multiphase Flow, 1998. 24(3): p. 387-410.

${ }^{33}$ Chorin, A.J., Numerical Solution of the Navier-Stokes Equations. Mathematics of Computation, 1968. 22(104): p. $745-762$.

${ }^{34} \mathrm{Kim}$, J. and P. Moin, Application of a fractional-step method to incompressible Navier-Stokes equations. Journal of Computational Physics, 1985. 59: p. 308-323.

${ }^{35}$ Singh, R., Three-dimensional marker-based multiphase flow computation using adaptive cartesian grid techniques, in Mechanical and Aerospace Engineering. 2006, University of Florida: Gainesville. p. 144.

${ }^{36}$ Engquist, B., A.K. Tornberg, and R. Tsai, Discretization of Dirac delta functions in level set methods. Journal of Computational Physics, 2005. 207(1): p. 28-51.

${ }^{37}$ Stockie, J.M., Analysis and Computation of Immersed Boundaries, with Application to Pulp Fibres, in Institute of Applied Mathematics. 1997, University of British Columbia.

${ }^{38}$ Singh, R., E. Uzgoren, W. Shyy, and M. Garbey. Three-Dimensional Adaptive, Cartesian Grid Method for Multiphase Flow Computations. in 43rd AIAA Aerospace Sciences Meeting and Exhibit. 2005. Reno, NV.

${ }^{39}$ Wang, Z. and Z.J. Wang, The Level Set Method on Adaptive Cartesian Grid For Interface Capturing. AIAA Paper No, 2004. 82 .

${ }^{40}$ Francois, M. and W. Shyy, Micro-scale drop dynamics for heat transfer enhancement. Progress in Aerospace Sciences, 2002. 38(4): p. 275-304.

${ }^{41}$ de Sousa, F.S., N. Mangiavacchi, L.G. Nonato, A. Castelo, M.F. Tomé, V.G. Ferreira, J.A. Cuminato, and S. McKee, A front-tracking/front-capturing method for the simulation of $3 D$ multi-fluid flows with free surfaces. Journal of Computational Physics, 2004. 198(2): p. 469-499.

${ }^{42}$ Al-Rawahi, N. and G. Tryggvason, Numerical simulation of dendritic solidification with convection: two-dimensional geometry. Journal of Computational Physics, 2002. 180(2): p. 471-496.

${ }^{43}$ Al-Rawahi, N. and G. Tryggvason, Numerical simulation of dendritic solidification with convection: Three-dimensional flow. Journal of Computational Physics, 2004. 194(2): p. 677-696.

${ }^{44}$ Shin, S. and D. Juric, Modeling three-dimensional multiphase flow using a level contour reconstruction method for front tracking without connectivity. Journal of Computational Physics, 2002. 180(2): p. 427-470.

${ }^{45}$ Patankar, S.V., Numerical Heat Transfer and Fluid Flow. 1980: McGraw-Hill.

${ }^{46}$ Shyy, W., Computational modeling for fluid flow and interfacial transport. 1994: Elsevier New York.

${ }^{47}$ Dussan, V.E.B., The moving contact line: slip boundary conditions. Journal of Fluid Mechanics, 1977. 77: p. 665-684.

${ }^{48}$ Hocking, L.M., A moving interface on a rough surface. Journal of Fluid Mechanics, 1976. 76: p. 801-817.

${ }^{49}$ Huang, H., D. Lian, and B. Wetton, Computation of a moving drop/bubble on a solid surface using a front tracking method. Communications in Mathematical Physics, 2004. 2(4): p. 535-552. 
${ }^{50}$ Fujioka, H. and J. Grotberg, Steady liquid plug flow in a 2-d channel. Faseb Journal, 2003. 17(4): p. A87-A87.

${ }^{51}$ Fujioka, H. and J.B. Grotberg, Steady Propagation of a Liquid Plug in a Two-Dimensional Channel. Journal of Biomechanical Engineering, 2004. 126: p. 567.

${ }^{52}$ Zheng, Y., H. Fujioka, J.C. Grotberg, and J.B. Grotberg, Effects of Inertia and Gravity on Liquid Plug Splitting at a Bifurcation. Journal of Biomechanical Engineering, 2006. 128: p. 707.

${ }^{53}$ Jiang, X. and A.J. James, Numerical simulation of the head-on collision of two equal-sized drops with van der Waals forces. Journal of Engineering Mathematics, 2007. 59(1): p. 99-121.

${ }^{54}$ Erneux, T. and S.H. Davis, Nonlinear rupture of free films. Physics of Fluids A: Fluid Dynamics, 1993. 5: p. 1117.

${ }^{55}$ Veldman, A.E.P., J. Gerrits, R. Luppes, J.A. Helder, and J.P.B. Vreeburg, The numerical simulation of liquid sloshing on board spacecraft. Journal of Computational Physics, 2007. 224(1): p. 82-99.

${ }^{56}$ Symons, E.P., Draining Characteristics of Hemispherically Bottomed Cylinders in a Low-Gravity Environment. 1978. 1970.

${ }^{57}$ Berenyi, S.G., Vapor ingestion phenomenon in hemispherically bottomed tanks in normal gravity and in weightlessness.

${ }^{58}$ Toole, L.E. and L.J. Hasting, An Experimental Study of the Behavior of a Sloshing Liquid Subjected to a Sudden Reduction in Acceleration. 1968, NASA. 